\title{
A GEOMETRIC APPROACH TO THE LINEAR PENROSE TRANSFORM ${ }^{1}$
}

BY

\author{
I. B. PENKOV
}

\begin{abstract}
We show that under a certain cohomological condition the theorem of Witten, Isenberg, Yasskin and Green about the inverse Penrose transform of a (non-self-dual) connection $\nabla$ (together with Manin's description of its curvature $\left.F_{\Gamma}\right)$ is true in a quite general situation. We then present a (multidimensional) version of the Penrose transform of a vector bundle in the language of jets. This gives a coordinate-free interpretation of certain results of Henkin and Manin, coding a number of classical field equations in terms of obstructions to infinitesimal extension of cohomology classes.
\end{abstract}

Introduction. In the last fifteen years the ideas of Roger Penrose (see for instance [22]) generated a new direction in mathematical physics - the twistor approach to field theory. The mathematical kernel of this theory (the Penrose transform) is the interpretation of a number of fundamental physical equations in space-time in terms of holomorphic geometrical objects on a so-called twistor space. This approach led to a number of remarkable results, for example to the solution of the instanton and monopole problems (see [2, 1, 12 and 13]). The large amount of literature devoted to the Penrose transform concentrates mainly on some special cases (the self-dual or non-self-dual case) and considers concrete equations of physical interest, such as the self-duality equation, the Yang-Mills equation, the Dirac and Laplace equations, etc. (see, for instance, $[1,2,6,10,14,15,18,19,24,25,26,28,30]$ ).

The aim of the present paper is to show that it is reasonable to consider this transform in a more general (multidimensional) situation. We look at the Penrose transform as a mechanism for processing purely geometrical information about coherent sheaves (or locally free sheaves) into information about differential operators. (In this paper we restrict ourselves to the case of linear differential equations.) This can be very useful in both directions: on the one hand, one can compute cohomology solving differential equations (which is not quite new in analytic geometry) and, on the other hand, one can study differential equations using analytic (or even algebraic) geometry (which is a new, extremely useful approach in the theory of differential equations).

Received by the editors August 18, 1983 and, in revised form, March 18, 1984.

1980 Mathematics Subject Classification. Primary 14F05, 53B15, 58A20.

${ }^{1}$ This paper is devoted to Vladimir Mikhailovich Tikhomirov on the occasion of his 50th birthday.

1985 American Mathematical Society $0002-9947 / 85 \$ 1.00+\$ .25$ per page 
The notion of a "Penrose transform" has not yet reached a definitive form (as opposed, for instance, to the Fourier transform), and our paper does not solve this problem.

We shall deal with the simplest geometrical mechanisms which unify and explain some of the low-dimensional results. (Some recent papers consider supermanifolds instead of manifolds (see [19 and 31]), but we restrict ourselves to usual complex manifolds and analytic spaces. We also do not discuss real structures.)

Let us briefly sketch the contents of the paper.

In $\S 1$ we present a generalized version of a result of J. Isenberg, Ph. Yasskin and P. Green [15] and E. Witten [27], transforming a connection $\nabla$ in a vector bundle $E$ on a manifold $\mathscr{U}$ into a vector bundle $E_{\mathscr{X}}$ on a certain manifold $\mathscr{X}(\mathscr{U})$ consisting of curves on $\mathscr{U}$. Then we discuss the corresponding variant of a theorem of Manin (see [30]), which gives a cohomological description of the curvature $F_{\nabla}$ of $\nabla$ in terms of the cohomology of the bundle $E_{\mathscr{C}}$ on $\mathscr{X}(\mathscr{U})$. The results of $\S 1$ were obtained in joint work with Yu. I. Manin. A special case was treated in [20].

In $\$ 2$ we present in the language of jet-bundles a more general geometrical variant of the procedure of reconstructing the pair $(E, \nabla)$ in terms of the vector bundle $E_{\mathscr{X}}$.

In $\S 3$ we explain geometrically a construction of Henkin and Manin [10], who showed that certain infinitesimal prolongations of the bundle $E_{\mathscr{X}}$ can carry new, additional information about linear differential equations on $\mathscr{U}$. The approach of $\S 2$ turns out to be useful here and allows us to obtain coordinate free proofs of the results of Henkin and Manin. The results of $\S \S 2$ and 3 were announced in [32].

I would like to emphasize that the present paper was written in communication with and under the influence of Professor Yu. I. Manin, to whom I am greatly indebted.

\section{An equivalence of categories.}

1.1. In this section we deal with the inverse Penrose transform of a connection, or more exactly of a pair $(E, \nabla)$, where $E$ is a vector bundle and $\nabla$ a connection on it. Proving that this is actually an equivalence of categories, we describe also the Penrose transform of an $\mathscr{U}$-trivial vector bundle. First we introduce the notion of an $X$-structure on a manifold. This is the geometric base on which our constructions take place.

Definition. Let $\mathscr{U}$ be a complex manifold, $\operatorname{dim}_{\mathrm{C}} \mathscr{U}=n$.

An $X$-structure on $\mathscr{U}$ is a commutative diagram

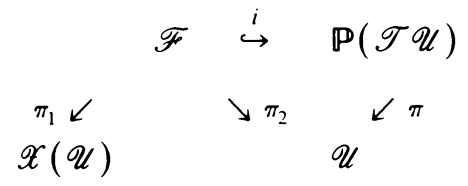

where $\mathscr{F}$ and $\mathscr{U}$ are complex manifolds, $\mathbb{P}(\mathscr{T} \mathscr{U})$ is the projectivization of the tangent bundle of $\mathscr{U}$ with its natural projection $\pi$, and $i, \pi_{1}, \pi_{2}$ are morphisms of complex manifolds with the properties:

$\mathrm{X} 1 . \pi_{2}$ is a smooth morphism. 
X2. $\pi_{1}$ is a fibration with connected one-dimensional fibers, transversal to the fibers of $\pi_{2}$.

$\mathrm{X} 3 . i$ is a closed embedding.

X4. $\forall u \in \mathscr{U}$ the map $\pi_{1}: \pi_{2}^{-1}(u) \rightarrow \mathscr{X}(\mathscr{U})$ is a (closed $\left.^{2}\right)$ embedding and $\forall x \in$ $\mathscr{X}(\mathscr{U})$ the map $\pi_{2}: \pi_{1}^{-1}(x) \rightarrow \mathscr{U}$ is an embedding.

EXAMPles of $X$-STRUCTURES. 1. Projective connection. If $\mathscr{U}$ satisfies some natural conditions (it must be strongly geodesically convex) a projective connection on $\mathscr{U}$ (see [16]) determines an $X$-structure, where $\mathscr{X}(\mathscr{U})$ is the manifold of geodesics of the projective connection, $\mathscr{F}$ coincides with $\mathbb{P}(\mathscr{T} \mathscr{U})$ and $i$ is the identity.

2. Conformal connection. A civilized conformal connection [16] on $\mathscr{U}$ determines an $X$-structure on $\mathscr{U}$, where $\mathscr{X}(\mathscr{U})$ is now the manifold of null-geodesics and $\mathscr{F}$ is the projectivization of the bundle of null-cones of the conformal structure.

3. Grassmann $X$-structure. Let $\mathscr{U} \subset \operatorname{Gr}(m, T)$ be an open submanifold (which may coincide with $\operatorname{Gr}(m, T))$ in the Grassmannian of $m$-planes in a complex linear space $T$. We have the diagram

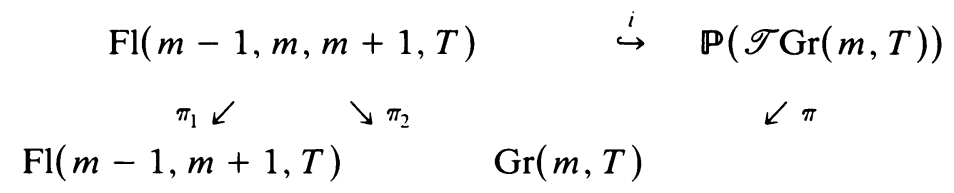

which, as it is easy to see, induces an $X$-structure on $\mathscr{U}: \mathscr{F}=\pi_{2}^{-1}(\mathscr{U}), \mathscr{X}(\mathscr{U})=$ $\pi_{1} \pi_{2}^{-1}(\mathscr{U})$. Here $i$ is the canonical embedding.

ReMARK. In fact, to determine an $X$-structure on $\mathscr{U}$ means to choose a class of (smooth) curves on $\mathscr{U}$ satisfying some natural conditions, one of which is that the set of curves passing through every point $u \in \mathscr{U}$ is a compact submanifold of fixed dimension of the projectivized tangent space (this manifold is identified with $\left.\pi_{2}^{-1}(u) \hookrightarrow \mathbb{P}\left(\mathscr{T}_{u} \mathscr{U}\right)\right)$. Then $\mathscr{X}(\mathscr{U})$ is naturally identified with the whole set of curves. Locally on $\mathscr{U}$ there always exist different $X$-structures.

Next we introduce a technical restriction on the $X$-structures which we shall work with in this paper.

DEFINITION. We call an $X$-structure admissible iff $\pi_{*} \mathscr{J}_{\mathscr{F}}(1)=R^{1} \pi_{*} \mathscr{J}_{\mathscr{F}}(1)=0$, where $\mathscr{J}_{\mathscr{F}}$ is the sheaf of ideals of $\mathscr{F}$ in $\mathbb{P}(\mathscr{T} \mathscr{U}), \mathscr{J}_{\mathscr{F}}(1)=\mathscr{J}_{\mathscr{F}} \otimes \mathcal{O}(1), \mathcal{O}(1)$ is the standard ample sheaf of Grothendieck on $\mathbb{P}(\mathscr{T} \mathscr{U})$ and $\pi_{*}$ is the direct image functor (see, for example, [9]) (the higher direct image functors we denote by $R^{i} \pi_{*}$ ).

It is easy to check that all three examples are examples of admissible $X$-structures.

1.2. Now we introduce the main objects that we shall deal with in $\$ 1$.

Fix an admissible $X$-structure on $\mathscr{U}$. Consider vector bundles $E$ on $\mathscr{U}$ equipped with a holomorphic connection $\nabla: E \rightarrow E \otimes \Omega^{1} \mathscr{U}$ (by $\Omega^{k} \mathscr{U}$ we denote the sheaves of holomorphic $k$-forms on $\mathscr{U}$ ) with the following property:

(i) The monodromy of $\nabla$ is zero on all curves of the type $\pi_{2} \pi_{1}^{-1}(x)$, where $x \in \mathscr{X}(\mathscr{U})$.

${ }^{2}$ If $\pi_{1}: \pi_{2}^{-1}(u) \rightarrow \mathscr{X}(\mathscr{U})$ is an embedding, it is necessarily a closed embedding (cf. [9, II Example 4.4]). 
It is standard to check that such pairs $(E, \nabla)$ form a category in which the morphisms of pairs are morphisms of bundles commuting with the connections.

REMARK. If the fibers of $\pi_{1}$ are simply connected (for the previous three examples this is locally always true) (i) is no restriction on $\nabla$.

It turns out that one can transform such pairs into vector bundles on $\mathscr{X}(\mathscr{U})$.

Definition. We call a vector bundle $E_{\mathscr{X}}$ on $\mathscr{X}(\mathscr{U}) \mathscr{U}$-trivial (or a Witten-IsenbergYasskin-Green bundle-see [3]) iff $E_{\mathscr{X}}$ is trivial on $\mathscr{X}(u):=\pi_{1} \pi_{2}^{-1}(u) \forall u \in \mathscr{U}$.

The following theorem describes simultaneously the Penrose transform of a $\mathscr{U}$-trivial bundle and its inverse.

TheOrem. Consider an admissible $X$-structure on $\mathscr{U}$. The category of pairs $(E, \nabla)$ on $\mathscr{U}$ satisfying the property (i) is equivalent to the category of $\mathscr{U}$-trivial vector bundles on $\mathscr{X}(\mathscr{U})$.

Sketch of Proof. We give only the constructions, because in the case of the four-dimensional $(\operatorname{dim} \mathscr{U}=4) \mathrm{Grassmann} X$-structure the theorem is proved in detail in [30] and the case of an arbitrary Grassmann $X$-structure is considered in [20]. The proof of the general case follows the same lines.

Consider the following diagram:

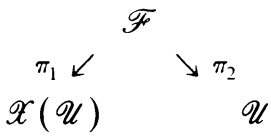

If we have a pair $(E, \nabla)$ on $\mathscr{U}$ we can first construct a pair $\left(E_{\mathscr{F}}, \nabla_{\pi_{1}}\right)$, where $E_{\mathscr{F}}$ is a vector bundle on $\mathscr{F}$ and $\nabla_{\pi_{1}}$ is a $\pi_{1}$-relative connection: $\nabla_{\pi_{1}}: E_{\mathscr{F}} \rightarrow E_{\mathscr{F}} \otimes \Omega_{\pi_{1}}^{1}\left(\Omega_{\pi_{1}}^{1}\right.$ being the sheaf of $\pi_{1}$-relative differential forms, which is an invertible sheaf on $\mathscr{F}$ ). This can be done as follows: let $E_{\mathscr{F}}=\pi_{2}^{*} E$ and let $\nabla_{\pi_{1}}$ be the composition of the pull-back $\nabla^{*}$ of $\nabla$, and the restriction to $\pi_{1}$-vertical forms:

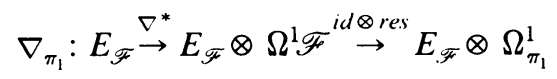

( res denotes restriction from $\Omega^{1} \mathscr{F}$ to $\Omega_{\pi_{1}}^{1}$ ). Evidently $\nabla_{\pi_{1}}$ has no monodromy on the fibers of $\pi_{1}$.

Further, we can transform the pair $\left(E_{\mathscr{F}}, \nabla_{\pi_{1}}\right)$ in a standard way into a vector bundle $E_{\mathscr{X}}$ on $\mathscr{X}(\mathscr{U})$. A more general construction is contained in [5]. Accordingly, the fiber of $E_{\mathscr{X}}$ at a point $x \in \mathscr{X}(\mathscr{U})$ is defined by

$$
E_{\mathscr{X}, x}:=\left\{\nabla_{\pi_{1}} \text {-horizontal sections of the bundle }\left.E_{\mathscr{F}}\right|_{\pi_{1}^{-1}(x)}\right\} .
$$

(Note that the fibers of $\pi_{1}$ are one-dimensional and $\nabla_{\pi_{1}}$ has no curvature on them; because its monodromy is also zero, the dimension of $E_{\mathscr{X}, x}$ as a $\mathrm{C}$-vector space is equal to the rank of the bundle $E$.) The triviality of $E_{\mathscr{C}}$ on $\mathscr{X}(u) \forall u \in \mathscr{U}$ in our situation follows from the triviality of $E_{\mathscr{F}}$ on the fibers of $\pi_{2}$.

Let us now reconstruct $(E, \nabla)$ given $E_{\mathscr{X}}$ on $\mathscr{X}(\mathscr{U})$. In [5] Deligne proves that the correspondence $\left(E_{\mathscr{F}}, \nabla_{\pi_{1}}\right) \leadsto E_{\mathscr{X}}$ is an equivalence of categories, thus knowing $E_{\mathscr{X}}$ we can reconstruct first the pair $\left(E_{\mathscr{F}}, \nabla_{\pi_{1}}\right)$. It is evident that $E_{\mathscr{F}}$ will be trivial on the fibers of $\pi_{2}$, so $E$ can be obtained just by putting $E:=\pi_{2 *} \pi_{1}^{*} E_{\mathscr{X}}$. It remains to 
reconstruct the connection $\nabla$. Here we use the admissibility condition and note that for an admissible $X$-structure we have the equality $\pi_{2 *} \Omega_{\pi_{1}}^{1}=\Omega^{1} \mathscr{U}$. Now we can define $\nabla$ just as the direct image ( $\pi_{2}$ is a projective morphism!) of the operator $\nabla_{\pi_{1}}$. Using the triviality of $\pi_{1}^{*} E_{\mathscr{X}}$ on the fibers of $\pi_{2}$ we have

$$
\nabla: E \rightarrow \pi_{2 *}\left(\pi_{1}^{*} E_{\mathscr{X}} \otimes \Omega_{\pi_{1}}^{1}\right)=E \otimes \Omega^{1} \mathscr{U} .
$$

One may easily check that this is a connection and that it coincides with out initial connection $\nabla$.

Note that it may happen that both categories under consideration will be trivial. This is, for instance, the case of a Grassmann $X$-structure with $\mathscr{U}=\operatorname{Gr}(m, T)$. As it is easy to prove [20], a vector bundle $E_{\mathscr{X}}$ on $\operatorname{Fl}(m-1, m+1, T)$, trivial on $\mathscr{X}(u)$ $\forall u \in \operatorname{Gr}(m, T)$, is itself trivial.

Another fact worth mentioning is that it follows from our description of the equivalence of categories that one can express the kernel of $\nabla$ directly in terms of $E_{\eta}$. Indeed, we have

$$
\operatorname{ker} \nabla=\{\nabla \text {-horizontal sections of } E\}=H^{0}\left(\mathscr{X}(\mathscr{U}), E_{\mathscr{X}}\right) .
$$

REMARK. This construction arose essentially from the ideas of $\mathrm{R}$. Penrose and R. Ward. At first the so-called self-dual case was studied (in connection with the equation of self-duality), where the role of $\mathscr{X}(\mathscr{U})$ was played by the manifold of null-surfaces ( $\alpha$-surfaces) in a four-dimensional conformally flat space-time, (see [ $\mathbf{1}$, 6, 18, 22, 24, 25, 28]). This led to the classification of instantons (see $[1,2,29])$. E. Witten, J. Isenberg, Ph. Yasskin and P. Green first proved the equivalence theorem in the four-dimensional flat non-self-dual case, or in our terminology, in the case of a four-dimensional Grassmann $X$-structure. They also proved more difficult theorems about the twistor transform of the (non-self-dual) Yang-Mills equation without sources [14, 15]. (Yu. I. Manin generalized their idea about Yang-Mills with sources; see $[3,10,30]$.) Our construction is a generalization of the equivalence theorem based on the same geometrical ideas. The $X$-structure is not the most general situation in which a pair $(E, \nabla)$ can be encoded as a vector bundle $E_{\mathscr{X}}$, because, for example, the self-dual four-dimensional case is not included. The requirement for the fibers of $\pi_{1}$ to be one-dimensional makes it possible to encode connections with arbitrary curvature (see 1.3 and 2.4.2).

1.3. In the applications of the above Theorem we are often interested in differential equations on the curvature of $\nabla-F_{\nabla}$, and it is important to describe $F_{\nabla}$ directly in terms of $E_{\mathscr{X}}$. In this section we give such a description. The Theorem in 1.3.1 generalizes Manin's description of the curvature in the four-dimensional case [30]. We formulate also a related global procedure, reconstructing $F_{\nabla}$ as an operator $F_{\Gamma}: E \rightarrow E \otimes \Omega^{2} \mathscr{U}$.

1.3.1. "Pointwise" description of $F_{\nabla}$. Denote by $\mathscr{Z}^{(k)} \hookrightarrow \mathscr{Y}$ the $k$ th formal neighbourhood of a closed complex submanifold $\mathscr{Z}$ in a complex manifold $\mathscr{Y}$. It is well known that if $E_{\mathscr{Z}^{(k)}}$ is a vector bundle (locally free sheaf) on $\mathscr{Z}^{(k)}$, then the group $H^{1}\left(\mathscr{Z}, \mathscr{E} n d\left(\left.E_{\mathscr{Z}(k)}\right|_{\mathscr{Z}}\right) \otimes \mathbb{S}^{k+1} N_{\mathscr{Z} / y y}^{*}\right)$, where $N_{\mathscr{Z} / y y}^{*}$ is the conormal bundle of $\mathscr{Z}$ in $\mathscr{Y}$, acts transitively on the set of all extensions of $E_{\mathscr{Z}(k)}$ to $\mathscr{Z}^{(k+1)}$. If there exists 
an extension $E_{\mathscr{P}^{(h+1)}}^{0}$ of $E_{\mathscr{P}^{(h)}}$, such that the natural map $H^{0}\left(\mathscr{E}_{n} \boldsymbol{C}_{\mathscr{P}^{(h+1)}}^{0}\right) \rightarrow$ $H^{()}\left(\mathscr{E}_{n d} \mathscr{E}_{\mathscr{P}^{(h)}}\right)$ is surjective, then the action is also effective. If, furthermore, $E_{\mathscr{P}^{(h)}}$ is the trivial bundle, then the set of all extensions is naturally identified with $H^{1}\left(\mathscr{Z}, \mathscr{E} n d\left(\left.E_{\mathscr{Z}^{(k)}}\right|_{\mathscr{Z}}\right) \otimes \mathbb{S}^{k+1} N_{\mathscr{Z} / \mathscr{Y}}^{*}\right)$. In the latter case every extension $E_{\mathscr{Z}^{(k+1)}}$ of $E_{\mathscr{P}^{(k)}}$ is characterized by an element

$$
\operatorname{cl}\left(E_{\mathscr{Z}^{(k+1)}}\right) \in H^{1}\left(\mathscr{Z}, \mathscr{E} n d\left(\left.E_{\mathscr{Z}^{(k)}}\right|_{\mathscr{Z}}\right) \otimes \mathbb{S}^{k+1} N_{\mathscr{Z} / \mathscr{y}}^{*}\right),
$$

which we call the Griffiths class of $E_{\mathscr{Q}^{(k+1)}}$ (see [8, 30 or 3]).

TheOREM. Let our $X$-structure be admissible and let $H^{1}\left(\mathscr{X}(u), \mathcal{O}_{\mathscr{X}(u)}\right)=0 \forall u \in \mathscr{U}^{\prime}$, $\mathscr{U}^{\prime}$ being an open set in $\mathscr{U}$. Then $\left.\forall u \in \mathscr{U}^{\prime} E_{\mathscr{X}}\right|_{\mathscr{X}_{(u)}^{(1)}}$ is trivial and there exists a canonical isomorphism $\theta_{u}:\left(\Omega^{2} \mathscr{U}\right)_{u} \otimes$ End $E_{u} \simeq H^{1}\left(\mathscr{X}^{(u)}(u), \mathscr{E}_{n d}\left(\left.E_{\mathscr{X}}\right|_{\mathscr{X}(u)}\right) \otimes \mathbb{S}^{2} N_{\mathscr{X}(u) / \mathscr{X}(\mathscr{U})}^{*}\right)$ such that $\theta_{u}\left(F_{\nabla, u}\right)=\operatorname{cl}\left(\left.E_{\mathscr{X}}\right|_{\mathscr{X}_{(u)}^{(2)}}\right)$, where $F_{\nabla}$ is represented as a section of the vector bundle $\Omega^{2} \mathscr{U} \otimes \mathscr{E}$ nd $E$ on $\mathscr{U}$.

This Theorem gives an explicit pointwise description of $F_{\nabla}$, transforming $F_{\nabla, u}$ into a natural "second-order" invariant of $E$, the Griffiths class of $\left.E_{\mathscr{X}}\right|_{\mathscr{X}_{(i)}^{(2)}}$.

Sketch of Proof. Yu. I. Manin proposed to interpret this theorem as an infinitesimal variant of the inverse Penrose transform of a pair $(E, \nabla)$. Let us consider the diagram of second-order formal neighbourhoods

$$
\begin{aligned}
& \overline{\mathscr{X}}_{(u)}^{(2)} \\
& \begin{array}{ll}
\pi_{1}^{(2)} \swarrow & \searrow \pi_{2}^{(2)} \\
\mathscr{X}_{(u)}^{(2)} & u^{(2)}
\end{array}
\end{aligned}
$$

where $\overline{\mathscr{X}}_{(u)}^{(2)}$ denotes the second formal neighbourhood of $\pi_{2}^{-1}(u)$ in $\mathscr{F}$, and $\mathscr{X}^{(2)}(u)$ and $u^{(2)}$ are the second neighbourhoods of $\mathscr{X}(u)$ and $u$ in $\mathscr{H}(\mathscr{U})$ and $\mathscr{U}$, correspondingly. It turns out that there exists a suitable notion of a connection in a vector bundle $E_{u^{(2)}}$ on $u^{(2)}\left(u^{(2)}\right.$ is just a point with nilpotents!), so that an equivalence theorem will be true.

Definition. Let $E_{u^{(2)}}$ be a vector bundle on $u^{(2)}$ (just a free $\mathcal{O}_{u^{(2)}}$-module of finite rank). Call a 1-connection in $E_{u^{(2)}}$ a C-linear mapping $\nabla_{1}:\left.E_{u^{(2)}} \rightarrow E_{u^{(2)}} \otimes\left(\Omega^{1} \mathscr{U}\right)\right|_{u^{(1)}}$, satisfying the Leibniz rule, i.e. if $e$ is a section of $E_{u^{(2)}}$ and $f$ is a function (section of $\left.\mathcal{O}_{1(2)}\right)$, then

$$
\nabla_{1}(f e)=f \cdot \nabla_{1}(e)+e \otimes d f
$$

(note that $\left.d f \in \Gamma\left(\left.\left(\Omega^{1} \mathscr{U}\right)\right|_{u^{(1)}}\right) !\right)$.

REMARK. In our special case the notion of a 1-connection is a convenient modification of the general notion of a connection on an analytic space (see [3]).

Now we can formulate

Statement 1 . For any $X$-structure, the functor $(E, \nabla) \leadsto E_{\mathscr{X}}$ from 1.2 induces a functor from the category of pairs $\left(E_{u^{(2)}}, \nabla_{1}\right)$ into the category of vector bundles

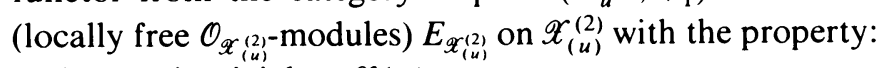

(j) $E_{\mathscr{X}_{(u)}^{(2)}}$ is trivial on $\mathscr{X}(u)$.

The foregoing statement says that the knowledge of the 1-connection $\nabla_{1}$ in $\left.E\right|_{u^{(2)}}$

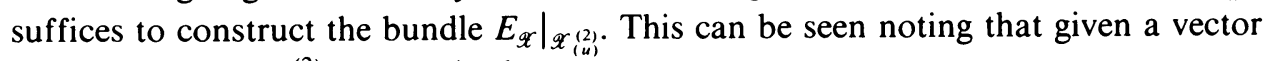
bundle $E_{\mathscr{X}_{(u)}^{(2)}}$ on $\mathscr{X}_{(u)}^{(2)}$, we obtain the exact sequence

$$
\left.0 \rightarrow E_{\mathscr{X}_{(u)}^{(2)}} \rightarrow \pi_{1}^{(2) *} E_{\mathscr{X}_{(u)}^{(2)}} \rightarrow \pi_{1}^{(2) *} E_{\mathscr{X}_{(u)}^{(2)}} \otimes\left(\Omega_{\pi_{1}}^{1}\right)\right|_{\overline{\mathscr{X}}_{(u)}^{(1)}} \rightarrow 0
$$


where the left arrow is the canonical embedding corresponding to $\pi_{1}^{(2)}$ and the right arrow is a $\pi_{1}^{(2)}$-relative 1-connection in $\pi_{1}^{(2) *} E_{\mathscr{X}_{(u)}^{(2)} \text {. (The definition of a relative }}$ 1 -connection is analogous to the absolute case.) Going backwards now, first one has to show that the pull-back of $\nabla_{1}$ determines a $\pi_{1}^{(2)}$-relative 1-connection in $\pi_{2}^{(2) *}\left(E_{u^{(2)}}\right)$, and then one can define $E_{\mathscr{X}_{(u)}^{(2)}}$ just as the kernel of this 1-connection. It is evident that $(j)$ is satisfied.

Having established this fact one notes that because our functor is purely infinitesimal it can be considered just as a map of $\mathbf{C}$-vector spaces:

Statement 2. A pair $\left(E_{u^{(2)}}, \nabla_{1}\right)$ is uniquely determined by the element $F_{\nabla_{1}} \in$ $\left(\Omega^{2} \mathscr{U}\right)_{u} \otimes$ End $E_{u}$, defined as the curvature of $\nabla_{1}$, and if, furthermore, our $X$-structure is admissible and $H^{1}\left(\mathscr{X}(u), \mathcal{O}_{\mathscr{X}(u)}\right)=0 \forall u \in \mathscr{U}^{\prime}$, the Griffiths class of a locally free sheaf $E_{\mathscr{X}_{(u)}^{(2)}}$ on $\mathscr{X}_{(u)}^{(2)}$, trivial on $\mathscr{X}(u)$, is correctly defined and determines $E_{\mathscr{X}_{(u)}^{(2)}}$ up to isomorphism.

The first part is an observation based on a local computation, which shows that using gauge transformations (which do not change the connection $\nabla_{1}$ ) we can represent the matrix of $\nabla_{1}$ in a standard form, for which the statement is evident. In some trivialization of $E_{u^{(2)}}$ let the matrix of $\nabla_{1}$ be $A_{0}+A_{1}$, where $A_{0}$ is a matrix of differential forms with constant coefficients and $A_{1}$ is a matrix of forms with linear coefficients. It turns out that there always exists a trivialization in which our matrix has the form $A=\sum A(i) d u^{i}$, where $A(i)=\sum A_{i j} u^{j}$ ( $A_{i j}$ are constant matrices) with $A_{i j}=0$ for $i \geqslant j$. But the curvature $F_{\nabla_{1}}$, equal to $d A+A \wedge A$, in our case reduces to $d A$ because the term $A \wedge A$ is a purely quadratic term. Now it is clear that in this case $d A$ determines $A$ in a unique way.

The second part follows from the fact that the admissibility of our $X$-structure and the condition $H^{1}\left(\mathscr{X}(u), \mathcal{O}_{\mathscr{X}(u)}\right)=0 \forall u \in \mathscr{U}^{\prime}$ gives $H^{1}\left(\mathscr{X}(u), N_{\mathscr{X}(u) / \mathscr{X}(\mathscr{U})}^{*}\right)=0 \forall u$ $\in \mathscr{U}^{\prime}$, which implies that $\left.E_{\mathscr{X}_{(u)}^{(2)}}\right|_{\mathscr{X}_{(u)}^{(1)}}$ is just the trivial bundle. Using the trivality of

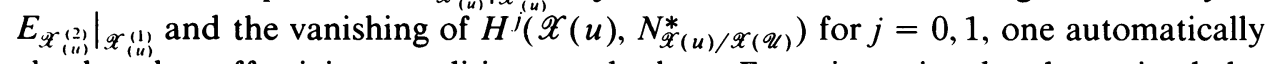
checks the effectivity condition, and thus $E_{\mathscr{X}_{(u)}^{(2)}}$ is uniquely determined by $\operatorname{cl}\left(E_{\mathscr{X}_{(i)}^{(2)}}\right)$, which is the "difference" between $E_{\mathscr{X}_{(u)}^{(2)}}$ and the trivial bundle in $H^{1}\left(\mathscr{X}(u), \mathscr{E} n d\left(\left.E_{\mathscr{X}_{(u)}^{(2)}}\right|_{\mathscr{X}(u)}\right) \otimes \mathbb{S}^{2} N_{\mathscr{X}(u) / \mathscr{X}(\mathscr{U})}^{*}\right)$.

In this way, for an admissible $X$-structure, such that $H^{1}\left(\mathscr{X}(u), \mathcal{O}_{\mathscr{X}(u)}\right)=0 \forall u \in$ $\mathscr{U}^{\prime}$, we can represent our functor as a map:

$$
\theta_{u}:\left(\Omega^{2} \mathscr{U}\right)_{u} \otimes \text { End } E_{u} \rightarrow H^{1}\left(\mathscr{X}(u), \mathscr{E} n d\left(\left.E_{\mathscr{X}}\right|_{\mathscr{X}(u)}\right) \otimes \mathbb{S} N_{\mathscr{X}(u) / \mathscr{X}(\mathscr{U})}^{*}\right)
$$

The last part is just a technical point. To prove that this is an isomorphism of C-vector spaces one shows that this map can be identified with the fiber of an isomorphism of vector bundles on $\mathscr{U}^{\prime}$. Consider the following exact sequence of bundles on $\pi_{2}^{-1}\left(\mathscr{U}^{\prime}\right) \hookrightarrow \mathscr{F}$ :

$$
0 \rightarrow N^{*} \rightarrow \pi_{2}^{*} \Omega^{1} \mathscr{U}^{\prime} \rightarrow \Omega_{\pi_{1}}^{1} \rightarrow 0
$$

where the right map is restriction to $\pi_{1}$-relative forms and $N^{*}$ is, by definition, the kernel, rk $N^{*}=n-1$. Its symmetric square has the form

$$
0 \rightarrow \mathbb{S}^{2} N^{*} \rightarrow \mathbb{S}^{2}\left(\pi_{1}^{*} \Omega^{1} \mathscr{U}^{\prime}\right) \rightarrow \pi_{2}^{*} \Omega^{1} \mathscr{U}^{\prime} \otimes \Omega_{\pi_{1}}^{1} \rightarrow 0
$$


and our theorem follows from the following technical statement:

Statement 3. Consider the direct image $R \pi_{2 *}$ of (2). It has the form

$$
0 \rightarrow 0 \rightarrow \mathbb{S}^{2} \Omega^{1} \mathscr{U}^{\prime} \rightarrow \Omega^{1} \mathscr{U}^{\prime} \otimes \Omega^{1} \mathscr{U}^{\prime} \rightarrow R^{1} \pi_{2 *} \mathbb{S}^{2} N^{*} \rightarrow \cdots
$$

and defines a canonical isomorphism

$$
\delta^{0}: \Omega^{1} \mathscr{U}^{\prime} \otimes \Omega^{1} \mathscr{U}^{\prime} / \mathbb{S}^{2} \Omega^{1} \mathscr{U}^{\prime} \rightarrow R^{1} \pi_{2 *} \mathbb{S}^{2} N^{*},
$$

such that $\forall u \in \mathscr{U}^{\prime}$

$$
\theta_{u}=\delta_{u}^{0} \otimes \mathrm{id}
$$

(Note that End $E_{u}=H^{0}\left(\mathscr{X}(u), \mathscr{E}\right.$ nd $\left.\left.E_{\mathscr{X}}\right|_{\mathscr{X}(u)}\right)$ and that Grauert's theorem [7] implies that $R^{1} \pi_{2 *}\left(\mathbb{S}^{2} \pi_{1}^{*} \Omega^{1} \mathscr{U}^{\prime}\right)=\mathbb{S}^{2} \Omega^{1} \mathscr{U}^{\prime} \otimes R^{1} \pi_{2 *} \mathcal{O}_{\pi_{2}}{ }^{\prime}\left(\mathscr{U}^{\prime}\right)=0$.)

REMARK. As a consequence of Statement 3 we obtain that for an admissible $X$-structure, with $H^{1}\left(\mathscr{X}(u), \mathcal{O}_{\mathscr{X}(u)}\right)=0 \forall u \in \mathscr{U}^{\prime}$ the functor from Statement 1 is an equivalence of categories.

Of course the importance of this theorem is in the explicit description of the curvature $F_{\nabla}$ on $\mathscr{X}(\mathscr{U})$ in purely geometric terms. But one may, for instance, derive the following geometric

COROllary. For an admissible X-structure, such that $H^{1}\left(\mathscr{X}(u), \mathcal{O}_{\mathscr{X}(u)}\right)=0 \forall u \in$ $\mathscr{U}^{\prime}$, every bundle on $\mathscr{X}_{(u)}^{(2)}$, trivial on $\mathscr{X}(u)$, can be extended to a bundle $E_{\mathscr{X}}$ on $\mathscr{X}\left(\mathscr{U}^{\prime \prime}\right)$, where $\mathscr{U}^{\prime \prime}$ is some neighbourhood of $u$.

Proof. The statement follows directly from the fact that it can be extended to a connection in the trivial bundle over some neighbourhood $\mathscr{U}^{\prime \prime}$ of $u$.

1.3.2. Global reconstruction of $F_{\nabla}$. Unfortunately we do not know a good global description of $F_{\nabla}$ on $\mathscr{X}(\mathscr{U})$, but our morphisms $\theta_{u}$ glue together $\mathscr{F}$. Consider the diagram

$$
\begin{array}{lc} 
& \mathscr{F}^{(2)} \hookrightarrow \mathscr{X}(\mathscr{U}) \times \mathscr{U} \\
p_{2} \swarrow & \searrow q_{2} \\
\mathscr{X}(\mathscr{U}) & \mathscr{U}
\end{array}
$$

where $\mathscr{F}^{(2)}$ is the second formal neighbourhood of $\mathscr{F}$ in $\mathscr{X}(\mathscr{U}) \times \mathscr{U}$. If now $R^{1} \pi_{2 *} \mathcal{O}_{\tilde{F}}=0$, then, as one can see (by the admissibility condition and the Leray spectral sequence), $H^{1}\left(\mathscr{F}, \mathscr{E} n d E_{\mathscr{F}} \otimes N^{*}\right)=0^{3}$ and thus $\left.\left(p_{2}^{*} E_{\mathscr{X}}\right)\right|_{\mathscr{F}^{(1)}}=\left.\left(q_{2}^{*} E\right)\right|_{\mathscr{F}(1)}$ as the unique extension of $E_{\mathscr{F}}$ to $\mathscr{F}^{(1)}$. This implies that the "difference" of $p_{2}^{*} E_{\mathscr{X}}$ and $q_{2}^{*} E$ defines an element $\operatorname{cl}\left(\left.p_{2}^{*} E_{\mathscr{X}}\right|_{\mathscr{F}(2)}\right) \in H^{1}\left(\mathscr{F}, \mathscr{E}\right.$ nd $\left.E_{\mathscr{F}} \otimes \mathbb{S}^{2} N^{*}\right)$ (one can note considering the vector bundle $q_{2}^{*} E$, that the effectivity condition is also satisfied) and we can prove the following globalization of our theorem on $\mathscr{F}$ :

Proposition 1. Let $\mathscr{U}$ be a Stein manifold and let $R^{1} \pi_{2 *} \mathcal{O}_{\mathscr{\pi}}=0$. We have a canonical isomorphism

$$
\theta: H^{0}\left(\mathscr{U}, \mathscr{E} n d E \otimes \Omega^{2} \mathscr{U}\right) \simeq H^{1}\left(\mathscr{F}, \mathscr{E} n d E_{\mathscr{H}} \otimes \mathbb{S}^{2} N^{*}\right)
$$

such that

$$
\theta\left(F_{\nabla}\right)=\operatorname{cl}\left(\left.p_{2}^{*} E_{\mathscr{Y}}\right|_{\widetilde{F}(2)}\right)
$$

\footnotetext{
${ }^{3}$ Where $N^{*}$ is now, by definition, the kernel of the restriction map $\pi_{2}^{*} \Omega^{1} \mathscr{U} \rightarrow \Omega_{\pi_{1}}^{1}$
} 
This theorem is not very satisfactory because, in principle, we would like to have an explicit global description of $F_{\nabla}$ on $\mathscr{X}(\mathscr{U})$ and not on $\mathscr{F}$. Although we do not know such a satisfactory construction, the next statement gives a possibility to reconstruct $F_{\nabla}$ as a linear operator on $\mathscr{U}$ directly in terms of $E_{\mathscr{X}}$. This is a generalization of a statement due to A. Beilinson (see [30, §7]).

Consider $p_{2}^{*} E_{\mathscr{X}}$ on $\mathscr{F}^{(2)}$ and assume again $R^{1} \pi_{2 *} \mathcal{O}_{\mathscr{F}}=0$. On $\mathscr{F}^{(2)}$ we have the exact sequence of $\mathcal{O}_{\mathscr{F}(2)}$-modules

$$
\left.0 \rightarrow E_{\mathscr{H}} \otimes \mathbb{S}^{2} N^{*} \rightarrow p_{2}^{*} E_{\mathscr{X}} \rightarrow\left(p_{2}^{*} E_{\mathscr{X}}\right)\right|_{\mathscr{F}(1)} \rightarrow 0
$$

Now consider the direct image $R q_{2 *}$ of this sequence.

Proposition 2. The functor $R q_{2}$ * transforms sequence (3) into the following sequence of $\mathcal{O}_{\mathscr{U}}$-modules:

$$
0 \rightarrow 0 \rightarrow \operatorname{ker} F_{\nabla} \rightarrow E \stackrel{\delta^{0}=F_{\ulcorner}}{\rightarrow} E \otimes \Omega^{2} \mathscr{U} \rightarrow \cdots
$$

where the coboundary morphism $\delta^{0}$ coincides with the curvature $F_{\nabla}$ considered as a linear operator:

$$
F_{\nabla}: E \rightarrow E \otimes \Omega^{2} \mathscr{U}
$$

The proof is a technical exercise, which we omit.

REMARK. In [3] N. Buchdahl gives an independent proof of Manin's results on $F_{\nabla}$ in the four-dimensional case. In the case of a Grassmann $X$-structure the theorem of 1.3.1 is announced in [20].

2. A geometric description of the direct image of $\nabla_{\pi_{1}}$. In this section we describe a generalization of the functor $E_{\mathscr{X}} \leadsto(E, \nabla)$. We present a geometric construction, which allows us to "push down" on $\mathscr{U}$ the relative connection $\nabla_{\pi_{1}}$ directly in terms of morphisms between jet-bundles.

2.1. First we replace the $X$-structures from $\S 1$ by diagrams

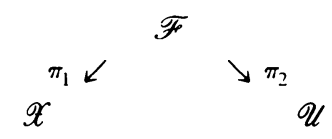

with the following properties [32]:

D1. $\pi_{1}$ and $\pi_{2}$ are surjective morphisms of complex manifolds with transversal fibers (with zero-dimensional intersection).

D2. $\pi_{1}$ is a smooth morphism and $\pi_{2}$ is a proper morphism.

D3. The map $\mathscr{F} \rightarrow \mathscr{X} \times \mathscr{U}, f \mapsto \pi_{1}(f) \times \pi_{2}(f)$ is a closed embedding.

These diagrams are more general and give new possibilities to explore the Penrose transform: if, for example, $\mathscr{U}$ is a flag space $\left(\mathcal{U}=\operatorname{Fl}\left(k_{1}, k_{2}, \ldots, k_{r}, T\right)\right.$ in a linear space $T$ ) already among the flag spaces in the same linear space $(T)$ there are different variants for the choice of $\mathscr{X}$.

We keep the symbol $\mathscr{X}$ for the "left-hand" space of diagram (D), but now write $\mathscr{X}$ alone (and not $\mathscr{X}(\mathscr{U})$ ) because $\mathscr{X}$ does not need to be determined naturally in terms of $\mathscr{U}$. The symbols $\mathscr{F}, E_{\mathscr{X}}$, etc. will also be used in a more general sense. 
2.2. The manifold $\mathscr{F}$ has two systems of infinitesimal extensions-the formal neighbourhoods of $\mathscr{F}$ in $\mathscr{F} \times \mathscr{U}$ and $\mathscr{X} \times \mathscr{U}$ :

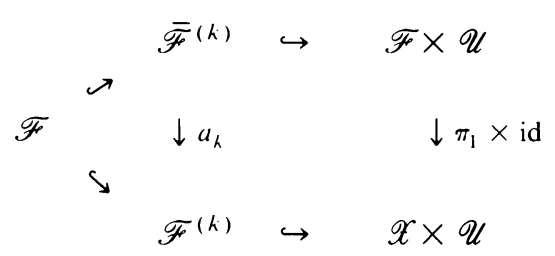

together with the surjection (of analytic spaces) $a_{k}: \overline{\mathscr{F}}^{(k)} \rightarrow \mathscr{F}^{(k)}$ ( $k$ as above denotes the order of the corresponding neighbourhood). The conormal bundle of $\mathscr{F}$ in $\mathscr{F} \times \mathscr{U}$ is canonically isomorphic to the bundle $\pi_{2}^{*} \Omega^{1} \mathscr{U}$. We denote the conormal bundle of $\mathscr{F}$ in $\mathscr{X} \times \mathscr{U}$ by $N^{*}$ as in $\S 1$ (the rank of $N^{*}$ may now be arbitrary). It is easy to see that we again have the exact sequence:

$$
0 \rightarrow N^{*} \rightarrow \pi_{2}^{*} \Omega^{1} \stackrel{\text { Ues }}{\rightarrow} \Omega_{\pi_{1}}^{1} \rightarrow 0
$$

because res is a surjective morphism of vector bundles by conditions D1 and D2.

We obtain the commutative diagram

$\left(D^{\prime}\right)$

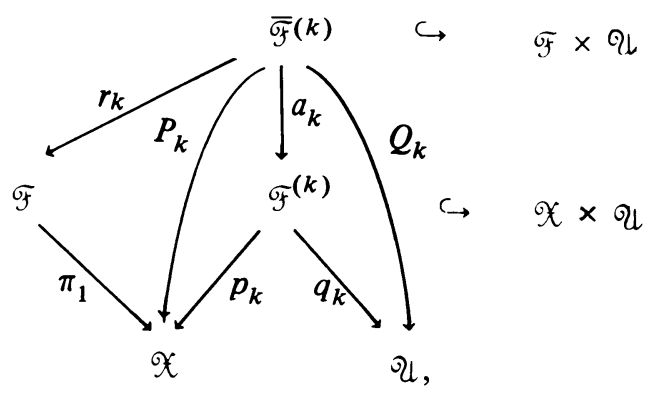

where $p_{k}, q_{k}, r_{k}$ are the restrictions of the natural projections and $P_{k}=a_{k}{ }^{\circ} p_{k}$, $Q_{h}=a_{h} \circ q_{h}$.

Now let $E_{\mathscr{X}}$ be a locally free sheaf of $\mathcal{O}_{\mathscr{X}}$ modules.

Consider the natural exact sequence of sheaves of abelian groups on $\mathscr{F}$ :

$$
0 \rightarrow \pi_{1}^{-1} E_{\mathscr{X}} \rightarrow \pi_{1}^{*} E_{\mathscr{X}} \stackrel{\nabla_{\pi_{1}}}{\rightarrow} \pi_{1}^{*} E_{\mathscr{X}} \otimes \Omega_{\pi_{1}}^{1},
$$

where $\nabla_{\pi_{1}}$ is the $\pi_{1}$-relative connection in $E_{\mathscr{X}}$. The "inverse image" of (4) on $\mathscr{F} \times \mathscr{U}$ evidently has the form

$$
0 \rightarrow\left(\pi_{1} \times \mathrm{id}\right)^{-1}\left(p^{*} E_{\mathscr{X}}\right) \rightarrow P^{*} \pi_{1}^{*} E_{\mathscr{X}} \stackrel{P^{*} \nabla_{\pi_{1}}}{\rightarrow} P^{*}\left(\pi_{1}^{*} E_{\mathscr{X}} \otimes \Omega_{\pi_{1}}^{1}\right),
$$

$P: \mathscr{F} \times \mathscr{U} \rightarrow \mathscr{F}$ and $p: \mathscr{X} \times \mathscr{U} \rightarrow \mathscr{X}$ denoting the corresponding projections.

Restricting now the bundle $P^{*} \pi_{1}^{*} E_{\mathscr{X}}$ to $\overline{\mathscr{F}}^{(k)}$ one may easily check that sequence (5) induces the following exact sequence:

$$
0 \rightarrow p_{k}^{*} E_{\mathscr{X}} \rightarrow P_{k}^{*} E_{\mathscr{X}} \stackrel{\nabla_{\pi_{1}}(k)}{\rightarrow} P_{k}^{*} E_{\mathscr{X}} \otimes_{\left.\mathcal{O}_{\mathscr{F}(k)}\right)} r_{k-1}^{*}\left(\Omega_{\pi_{1}}^{1}\right),
$$

which is, of course, an exact sequence of $\mathcal{O}_{\widetilde{F}(k)}$-modules. (The map $\nabla_{\pi_{1}}(k)$ is only $\mathcal{O}_{\tilde{F}^{\prime}(k)}$-linear and not $\mathcal{O}_{\overline{\bar{F}^{\prime}}(k)}$-linear, being the restriction of the relative connection 
$P^{*} \nabla_{\pi_{1}}$.) The direct image of this sequence under the morphism $q_{k}$ will be of main interest to us. $\left(P_{k}^{*} E_{\mathscr{T}}\right.$ and $P_{k}^{*} E_{\mathscr{X}} \otimes r_{k-1}^{*}\left(\Omega_{\pi_{1}}^{1}\right)$ have natural structures of $\mathcal{O}_{\overline{\mathscr{F}}(k) \text {-mod- }}$ ules, but their direct images of $\mathscr{U}$ via the morphisms $Q_{k}$ and $q_{k}$ obviously coincide.)

THEOREM. (a) $\forall k, l \geqslant 0$ we have the following canonical isomorphism:

$$
R^{\prime} Q_{k *} P_{k}^{*} E_{\mathscr{X}}=\mathscr{J}_{e} t^{k}\left(R^{\prime} \pi_{2 *} \pi_{1}^{*} E_{\mathscr{X}}\right) \text {. }
$$

(b) $\forall k, l \geqslant 0$ sequence (6) determines a canonical morphism of $\mathcal{O}_{\mathscr{U}}$-modules

$$
\Phi_{k-1}^{\prime}: \mathscr{J}_{e} t^{k}\left(R^{\prime} \pi_{2 *} \pi_{1}^{*} E_{\mathscr{X}}\right) \rightarrow \mathscr{J}_{e} t^{k-1}\left(R^{\prime} \pi_{2 *}\left(\pi_{1}^{*} E_{\mathscr{X}} \otimes \Omega_{\pi_{1}}^{1}\right)\right)
$$

and thus a linear differential operator of order $k$ :

$$
\tilde{\Phi}_{k-1}^{\prime}: R^{\prime} \pi_{2 *} \pi_{1}^{*} E_{\mathscr{X}} \rightarrow \mathscr{J}_{e} t^{k-1}\left(R^{\prime} \pi_{2 *}\left(\pi_{1}^{*} E_{\mathscr{X}} \otimes \Omega_{\pi_{1}}^{1}\right)\right) \text {. }
$$

(c) $\forall k \geqslant 2 \Phi_{k-1}^{\prime}$ are the $(k-1)$-prolongations of $\tilde{\Phi}_{0}^{\prime}$, i.e. the following diagram is commutative:

$$
\begin{array}{rrrrr}
\tilde{\Phi}_{0}^{\prime} & : & R^{\prime} \pi_{2 *} \pi_{1}^{*} E_{\mathscr{X}} & \rightarrow & R^{\prime} \pi_{2 *}\left(\pi_{1}^{*} E_{\mathscr{X}} \otimes \Omega_{\pi_{1}}^{1}\right) \\
\downarrow & \downarrow j_{h} & & & \downarrow j_{k-1} \\
\Phi_{k-1}^{\prime}: & \mathscr{J}_{e} t^{k}\left(R^{\prime} \pi_{2 *} \pi_{1}^{*} E_{\mathscr{X}}\right) & \rightarrow \mathscr{J}_{e} t^{k-1}\left(R^{\prime} \pi_{2 *}\left(\pi_{1}^{*} E_{\mathscr{X}} \otimes \Omega_{\pi_{1}}^{1}\right)\right)
\end{array}
$$

( by $j$, we denote the universal differential operator of order $r$ : see [23]).

2.3. Proof of The Theorem. (a) Let us prove the more general fact:

LEMMA 1. Let $E_{\mathscr{F}}$ be a coherent sheaf on $\mathscr{F}$. Then $\forall k, l \geqslant 0$

$$
R^{\prime} Q_{k} * r_{k}^{*} E_{\mathscr{F}}=\mathscr{J}_{e} t^{k}\left(R^{\prime} \pi_{2 *} E_{\mathscr{F}}\right)
$$

Proof. Consider the commutative diagram

$\left(D^{\prime \prime}\right)$

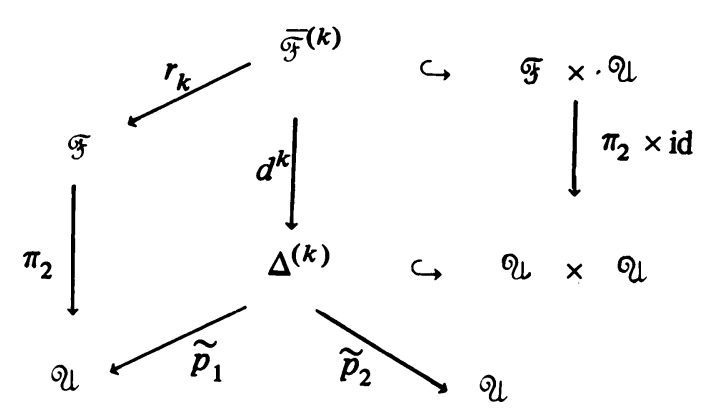

where $\Delta^{(k)}$ is the $k$ th formal neighbourhood of the diagonal $\Delta$ in $\mathscr{U} \times \mathscr{U}$. By definition for every coherent sheaf $E$ on $\mathscr{U}$ we have $\tilde{p}_{2 *} \tilde{p}_{1}^{*} E=\mathscr{J}_{e} t^{k} E$. Because we begin with a sheaf on $\mathscr{F}$, first we have to take its direct image on $\mathscr{U}-R^{\prime} \pi_{2 *} E_{\mathscr{F}}$ and 
then consider $\tilde{p}_{2 *} \tilde{p}_{1}^{*}\left(R^{\prime} \pi_{2 *} E_{\tilde{F}}\right)$. Thus our problem reduces to the canonical isomorphism of functors:

$$
\tilde{p}_{2 *} \circ \tilde{p}_{1}^{*} \circ R^{\prime} \pi_{2 *}=\tilde{p}_{2 *} \circ R^{l} d_{*}^{k} \circ r_{k}^{*} .
$$

Evidently it is sufficient to show that $\tilde{p}_{1}^{*} \circ R^{l} \pi_{2 *}=R^{l} d_{*}^{k} \circ r_{k}^{*}$. The main point is to note that $\overline{\mathscr{F}}^{(k)}=\mathscr{F} \times_{\mathscr{U}} \Delta^{(k)}$, and thus the part of the diagram, which is interesting to us, takes the form:

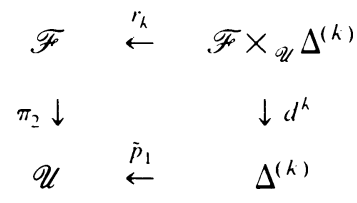

In this situation we always have a canonical morphism of functors

$$
\tilde{p}_{1}^{*} \circ R^{\prime} \pi_{2 *} \rightarrow R^{\prime} d_{*}^{k} \circ r_{k}^{*}
$$

because our diagram corresponds to the diagram of base extension according to $\tilde{p}_{1}$ : $\Delta^{(h)} \rightarrow \mathscr{U}$. But this base extension is flat (cf., for example, [9 III, §9]), thus our morphism of functors is an isomorphism. This completes the proof of the lemma.

Putting $E_{\mathscr{\mathscr { K }}}=\pi_{1}^{*} E_{\mathscr{X}}$ we obtain (a).

(b) From (a) we get

$$
\begin{aligned}
R^{\prime} q_{k *} P_{k}^{*} E_{\mathscr{X}}=R^{\prime} Q_{k *} P_{k}^{*} E_{\mathscr{C}} & =\mathscr{J}_{e} t^{k}\left(R^{\prime} \pi_{2 *} \pi_{1}^{*} E_{\mathscr{X}}\right), \\
R^{\prime} q_{k-1 *}\left(P_{k}^{*} E_{\mathscr{X}} \otimes r_{k-1}^{*}\left(\Omega_{\pi_{1}}^{1}\right)\right) & =R^{\prime} Q_{k-1 *}\left(P_{k}^{*} E_{\mathscr{X}} \otimes r_{k-1}^{*}\left(\Omega_{\pi_{1}}^{1}\right)\right) \\
& =\mathscr{J}_{e} t^{k-1}\left(R^{\prime} \pi_{2 *}\left(\pi_{1}^{*} E_{\mathscr{X}} \otimes \Omega_{\pi_{1}}^{1}\right)\right)
\end{aligned}
$$

and thus $\Phi_{h-1}^{l}$ can be defined as the direct image of the map $\nabla$ :

$$
\Phi_{k-1}^{\prime}=R^{\prime} q_{k *}(\nabla) \text {. }
$$

(c) Our statement means that all the information about $\Phi_{k-1}^{l}$ is contained in the differential operator $\tilde{\Phi}_{0}^{l}$. From the construction of $\tilde{\Phi}_{0}^{l}$ it follows easily that $\tilde{\Phi}_{0}^{l}$ is the map of sheaves of abelian groups

$$
R^{\prime} \pi_{2 *} \pi_{1}^{*} E_{\mathscr{X}} \rightarrow R^{\prime} \pi_{2 *}\left(\pi_{1}^{*} E_{\mathscr{X}} \otimes \Omega_{\pi_{1}}^{1}\right)
$$

induced by the map $\nabla_{\pi_{1}}$.

Thus we obtain a natural diagram

$$
\begin{aligned}
& \tilde{\Phi}_{0}^{\prime}: R^{\prime} \pi_{2 *} \pi_{1}^{*} E_{\mathscr{X}} \quad \rightarrow \quad R^{\prime} \pi_{2 *}\left(\pi_{1}^{*} E_{\mathscr{X}} \otimes \Omega_{\pi_{1}}^{1}\right) \\
& \downarrow \\
& \Phi_{k-1}^{\prime}: R^{\prime} q_{k *} P_{k}^{*} E_{\mathscr{X}} \quad \rightarrow \quad R^{\prime} q_{k-1 *}\left(P_{k}^{*} E_{\mathscr{X}} \otimes r_{k-1}^{*}\left(\Omega_{\pi_{1}}^{1}\right)\right) \\
& \|\quad\| \\
& \mathscr{J}_{e} t^{k}\left(R^{\prime} \pi_{2 *} \pi_{1}^{*} E_{\mathscr{X}}\right) \quad \mathscr{J}_{e} t^{k-1}\left(R^{\prime} \pi_{2 *}\left(\pi_{1}^{*} E_{\mathscr{X}} \otimes \Omega_{\pi_{1}}^{1}\right)\right)
\end{aligned}
$$


The fact that the vertical arrows of this diagram coincide with $j_{k}$ and $j_{k-1}$ follows from the following

LEMMA 2. Let $E_{\mathscr{T}}$ be a coherent sheaf on $\mathscr{F}$. The canonical morphism of sheaves of abelian groups $\Xi: R^{\prime} \pi_{2 *} E_{\mathscr{F}} \rightarrow R^{\prime} Q_{k} r_{k}^{*} E_{\mathscr{F}}=\mathscr{J}_{e} t^{k}\left(R^{\prime} \pi_{2 *} E_{\mathscr{F}}\right)$ induced by the diagram

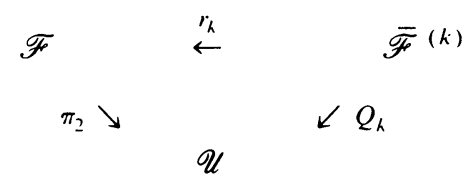

coincides with $j_{k}$.

Proof of THE LemMa. The statement follows again from the commutativity of diagram ( $\left(\mathrm{D}^{\prime \prime}\right)$. The canonical isomorphism of functors $\tilde{p}_{1}^{*} \circ R^{\prime} \pi_{2 *}=R^{\prime} d_{*}^{k} \circ r_{k}^{*}$ allows us to consider the mapping $R^{\prime} \pi_{2 *} E_{\mathscr{F}} \rightarrow \tilde{p}_{2 *} \tilde{p}_{2 *} R^{\prime} \pi_{2 *} E_{\mathscr{F}}$ instead of the mapping $R^{\prime} \pi_{2 *} E_{\mathscr{F}} \rightarrow \tilde{p}_{2 *} R^{\prime} d_{*}^{k} r_{k}^{*} E_{\mathscr{F}}$. But the former mapping coincides with $j_{k}$ by definition. The lemma is proved and this completes the proof of (c).

REMARK. The theorem gives a geometric point of view on the procedure of pushing down the relative connection in the vector bundle $\pi_{1}^{*} E_{\mathscr{X}}$. Of course, in the case of an admissible $X$-structure the operator $\tilde{\Phi}_{0}^{0}$ coincides with the connection $\nabla$. In order to obtain the full Penrose transform of $E_{\mathscr{X}}$ in the general case one clearly should consider the direct image of the whole $\pi_{1}$-relative de Rham complex $D R_{\pi_{1}}\left(\pi_{1}^{*} E_{\mathscr{X}}\right.$ ) of $\pi_{1}^{*} E_{\mathscr{X}}$ (for the self-dual case see [6]), but here we restrict ourselves to the description of the direct images of the relative connection itself.

2.4. EXAMPLES.

2.4.1. An example, which does not reduce to the case considered in $\$ 1$, is a generalization of the global Dirac operators on $\operatorname{Gr}(2,4)$ (see $[10,11]$ ). We consider the Grassmann $X$-structure, but in contrast to $\S 1$ begin with a bundle $E_{\mathscr{X}}$ which is not $\mathscr{U}$-trivial. So we have the diagram:

$$
\begin{gathered}
\operatorname{Fl}(m-1, m, m+1, T) \\
\pi_{1} \swarrow \quad \\
\operatorname{Fl}(m-1, m+1, T) \\
\operatorname{Gr}(m, T), \operatorname{dim} T=s .
\end{gathered}
$$

Put

$$
E_{\mathscr{X}}=\mathcal{O}(0,-s+m-1),
$$

where more generally $\mathcal{O}(i, j)$ denotes the invertible sheaf on $\operatorname{Fl}(m-1, m+1, T)$ induced by the canonical embedding of $\operatorname{Fl}(m-1, m+1, T)$ in $\operatorname{Gr}(m-1, T) \times$ $\operatorname{Gr}(m+1, T)$ from the sheaf $\left(\operatorname{det} S_{m-1}^{*}\right)^{\otimes i} \otimes\left(\operatorname{det} S_{m+1}^{*}\right)^{\otimes j}\left(S_{m-1}\right.$ and $S_{m+1}$ are the tautological bundles, and $S_{m-1}^{*}$ and $S_{m+1}^{*}$ are their duals). A straightforward computation using the explicit description of the cohomology of invertible sheaves on projective spaces (in the relative situation) shows that we obtain

$$
\Phi_{0}^{s-m-1}: \mathscr{J}_{e} t^{1}\left[\left(T / S_{m}\right) \otimes \Lambda^{s-m}\left(T / S_{m}\right)\right] \rightarrow S_{m} \otimes \Lambda^{s-m}\left(T / S_{m}\right)
$$

and it is easy to check that this operator is not identically zero:

$$
\operatorname{ker} \Phi_{0}^{s-m-1}=q_{1 *} p_{1}^{*} \mathcal{O}(0,-s+m+1) \neq \mathscr{J}_{e} t^{1}\left[\left(T / S_{m}\right) \otimes \Lambda^{s-m}\left(T / S_{m}\right)\right] .
$$


Putting alternatively $E_{\mathscr{T}}=\mathcal{O}(-m-1,0)$, we obtain

$$
\Phi_{0}^{m-1}: \mathscr{J}_{e} t^{1}\left[\Lambda^{m-1} S_{m} \otimes\left(\Lambda^{m} S_{m}\right)^{\otimes m+1}\right] \rightarrow\left(T / S_{m}\right)^{*} \otimes\left(\Lambda^{m} S_{m}\right)^{\otimes m} .
$$

As it is easy to see, $\tilde{\Phi}_{0}^{s-m-1}$ and $\tilde{\Phi}_{0}^{m-1}$ are $\operatorname{GL}(T)$ invariant operators (we begin with a $\operatorname{GL}(T)$-invariant bundle and our construction evidently preserves the group action). If $m=2$ and $s=4$ they coincide with the Dirac operators, but in the general case the role of the spinor bundles is played by $S_{m}$ and $T / S_{m}$.

Another pair of operators which may be obtained in this way are the Penrose (or twistor) operators. They correspond to the bundles $\mathcal{O}(0,1)$ and $\mathcal{O}(1,0)$ on $\mathrm{Fl}(m-1, m+1, T)$ and act as follows:

$$
\begin{aligned}
& \Phi_{0}^{0}: \mathscr{J}_{e} t^{1}\left(T / S_{m}\right)^{*} \rightarrow S_{m} \otimes \mathbb{S}^{2}\left(T / S_{m}\right)^{*}, \\
& \Phi_{0}^{0}: \mathscr{J}_{e} t^{1}\left(\Lambda^{m-1} S_{m}^{*}\right) \rightarrow\left(T / S_{m}\right)^{*} \otimes \mathbb{S}^{2}\left(S_{m}\right) \otimes \Lambda^{m} S_{m}^{*} .
\end{aligned}
$$

Tensoring our initial invertible sheaves on $\mathscr{X}$ with a bundle $E_{\mathscr{X}}$ corresponding to an Yang-Mills field on an open set $\mathscr{U} \subset \operatorname{Gr}(m, T)$ we obtain these operators on a "Yang-Mills background".

2.4.2. The operators $\tilde{\Phi}_{0}^{0}$ turn out to be connections not only for admissible $X$-structures. But if $\pi_{1}$ has relative dimension $>1$, these must be connections with restrictions on the curvature. For example, we have the following

Proposition. Consider the diagram

$$
\begin{array}{ccc} 
& \mathscr{F} \hookrightarrow & \operatorname{Fl}(k, l, T) \\
\operatorname{Gr}(k, T) \hookleftarrow \mathscr{X} & \searrow \pi_{2} \\
& & \mathscr{U} \hookrightarrow \operatorname{Gr}(l, T),
\end{array}
$$

where $\mathscr{U}$ is an open submanifold, $\mathscr{F}=\pi_{2}^{-1}(\mathscr{U})$ and $\mathscr{X}=\pi_{1} \pi_{2}^{-1} \mathscr{U}$. If $E_{\mathscr{X}}$ is $\mathscr{U}$-trivial (trivial on all submanifolds $\pi_{1} \pi_{2}^{-1}(u), u \in \mathscr{U}$ ) $\tilde{\Phi}_{0}^{0}$ is a connection, but if $k \neq l-1, \tilde{\Phi}_{0}^{0}$ is always a flat connection. If $k=l-1$, the curvature of $\tilde{\Phi}_{0}^{0}$ is a section of the bundle End $E \otimes \mathbb{S}^{2} S_{m} \otimes \Lambda^{2}\left(T / S_{m}\right)^{*}$.

Proof. Looking at the exact sequence

$$
0 \rightarrow N^{*} \rightarrow \pi_{2}^{*}\left(\Omega^{1} \mathscr{U}\right) \rightarrow \Omega_{\pi_{1}}^{1} \rightarrow 0
$$

we see by a direct computation that

$$
\pi_{2 *} \Omega_{\pi_{1}}^{1}=\Omega^{1} \mathscr{U}, \quad R^{i} \pi_{2 *} \Omega_{\pi_{1}}^{1}=0, \quad R^{i} \pi_{2 *} N^{*}=0, \quad \forall i>0 .
$$

Thus $\tilde{\Phi}_{0}^{0}$ is a first-order operator, acting from $E=\pi_{2 *} \pi_{1}^{*} E_{\mathscr{X}}$ to $E \otimes \Omega^{1} \mathscr{U}$ (here we use the $\mathscr{U}$-triviality of $E_{\mathscr{X}}$ !), i.e. $\Phi_{0}^{0}: \mathscr{J}_{e} t^{1} E \rightarrow E \otimes \Omega^{1} \mathscr{U}$, and one can see that $\tilde{\Phi}_{0}^{0}$ is a connection.

In this situation the curvature can be described as in 1.3.2 (Proposition 2) as a morphism

$$
F_{\nabla}: E \rightarrow E \otimes R^{1} \pi_{2 *} \mathbb{S}^{2} N^{*}
$$


But a simple cohomological computation shows that

$$
\begin{aligned}
& R^{1} \pi_{2 *} \mathbb{S}^{2} N^{*}=0 \quad \text { when } k \neq l-1, \\
& R^{1} \pi_{*} \mathbb{S}^{2} N^{*}=\mathbb{S}^{2}\left(T / S_{m}\right)^{*} \otimes \Lambda^{2} S_{m} \quad \text { when } k=l-1,
\end{aligned}
$$

and this implies our statement.

Thus, in this situation if we like our bundle $E_{\mathscr{X}}$ to carry some information about the curvature of $\nabla$, we have to restrict ourselves only to the case $k=l-1$ which is a direct generalization of the usual twistor transform (see, for instance, [6]).

2.5. In some special cases it is possible to encode the kernels of the operators $\tilde{\Phi}_{0}^{l}$ (or equivalently $\tilde{\Phi}_{k-1}^{\prime} \forall k \geqslant 0$ ) directly in terms of the cohomology of $E_{\mathscr{X}}$ on $\mathscr{X}$. Suppose that our diagram (D) is a $X$-structure on $\mathscr{U}$. $\tilde{\Phi}_{0}^{l}$ is the direct image of the $\pi_{1}$-relative connection on $E_{\mathscr{F}}=\pi_{1}^{*} E_{\mathscr{X}}$ and if the Leray spectral sequence (under the morphism $\pi_{2}$ ) of $\pi_{1}^{*} E_{\mathscr{X}}$ and $\pi_{1}^{*} E_{\mathscr{X}} \otimes \Omega_{\pi_{1}}^{1}$ degenerates, we can encode $\tilde{\Phi}_{0}^{l}$ in terms of $\mathscr{F}$ :

$$
\begin{gathered}
\tilde{\Phi}_{0}^{\prime}: H^{\prime}\left(\mathscr{F}, E_{\mathscr{F}}\right) \rightarrow H^{\prime}\left(\mathscr{F}, E_{\mathscr{F}} \otimes \Omega_{\pi_{1}}^{1}\right) \\
\| \\
H^{0}\left(\mathscr{U}, R^{\prime} \pi_{2 *} E_{\mathscr{F}}\right) \rightarrow H^{0}\left(\mathscr{U}, R^{\prime} \pi_{2 *}\left(E_{\mathscr{F}} \otimes \Omega_{\pi_{1}}^{1}\right)\right) .
\end{gathered}
$$

If, further, the coboundary morphism $H^{l-1}\left(\mathscr{F}, E_{\mathscr{F}} \otimes \Omega_{\pi_{1}}^{1}\right) \rightarrow H^{\prime}\left(\mathscr{F}, \pi_{1}^{-1}\left(E_{\mathscr{X}}\right)\right)$ is zero (the vanishing of $H^{1-1}\left(\mathscr{F}, E_{\mathscr{F}} \otimes \Omega_{\pi_{1}}^{1}\right)$ is sufficient), then we obtain

$$
\operatorname{ker} \Phi_{0}^{\prime}=H^{\prime}\left(\mathscr{F}, \pi_{1}^{-1}\left(E_{\mathscr{X}}\right)\right) \text {. }
$$

In order to "push down" this isomorphism on $\mathscr{X}(\mathscr{U})$, we have to put some natural conditions on the morphism $\pi_{1}$. More exactly we have the following result [4]:

Proposition. If $H^{p}\left(\pi^{-1}(x), \mathbf{C}\right)=0 \forall x \in \mathscr{X}(\mathscr{U}) \forall p, 1 \leqslant p \leqslant l$, then for every bundle $E_{\mathscr{X}}$ the canonical morphism

$$
H^{\prime}\left(\mathscr{X}(\mathscr{U}), E_{\mathscr{X}}\right) \rightarrow H^{\prime}\left(\mathscr{F}, \pi_{1}^{-1}\left(E_{\mathscr{X}}\right)\right)
$$

is an isomorphism.

In this way we see that, in the case of an $X$-structure, if some natural vanishing conditions are satisfied, we obtain that $\operatorname{ker} \tilde{\Phi}_{\theta}^{\prime}=H^{\prime}\left(\mathscr{X}(\mathscr{U}), E_{\mathscr{X}}\right)$. (In the general case one should consider the whole complex $D R_{\pi_{1}}\left(\pi_{1}^{*} E_{\mathscr{X}}\right)$ and the possible degeneration of the corresponding spectral sequence.)

3. The construction of Henkin and Manin. Henkin and Manin [10, 11] showed that if the vector bundle $E_{\mathscr{X}}$ can be extended to certain infinitesimal extensions of $\mathscr{X}$, the extended vector bundle can carry new, nontrivial information about some linear differential operators on $\mathscr{U}$. More precisely, they considered the four-dimensional Grassmann $X$-structure

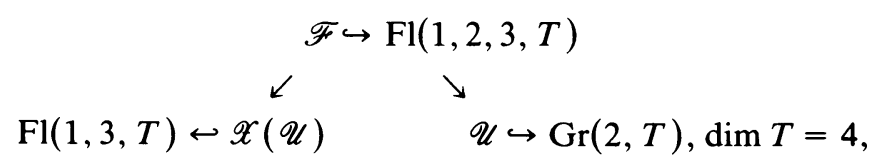


with the natural embeddings of $\mathscr{X}(\mathscr{U})$ in the formal neighbourhoods $\mathscr{X}(\mathscr{U})^{(k)}$ of $\mathscr{X}(\mathscr{U})$ in $\mathbb{P}(T) \times \mathbb{P}\left(T^{*}\right)$ and showed that the complex conformal Laplace and Dirac operators on $\mathscr{U}$ are closely connected with these formal neighbourhoods. They proved that the Laplace and Dirac operators can be represented as certain cohomological operators on $\mathscr{X}(\mathscr{U})^{(k)}, k=1,2$. (Henkin and Manin considered also the Yang-Mills equation, but as said above, we restrict ourselves to linear differential equations.) However, their proof was computational and it was not very clear what invariant geometric mechanism lies under these facts.

Here we show that the geometric poin ${ }^{+}$of view developed in $\$ 2$ allows us to give coordinate-free proofs of these results. The method consists in constructing some natural extensions of $\mathscr{F}$ and comparing their cohomological properties with the already known formal neighbourhoods of $\mathscr{F}$ in $\mathscr{F} \times \mathscr{U}$, which are responsible for the jet-bundles.

3.1. Consider again our diagram (D):

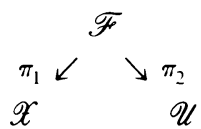

and suppose that we have two closed embeddings

$$
\mathscr{X} \hookrightarrow \mathscr{X}^{a} \hookrightarrow \mathscr{X}^{b}
$$

where $\mathscr{X}^{a}$ and $\mathscr{X}^{b}$ are some analytic spaces. (For example, $\mathscr{X}^{a}$ and $\mathscr{X}^{b}$ can be some formal neighbourhoods of $\mathscr{X}$ in a manifold $\hat{\mathscr{X}}$.) We obtain two more embeddings of $\mathscr{F}$

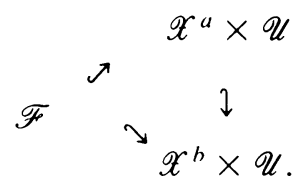

Denote the corresponding formal neighbourhoods of $\mathscr{F}_{\text {by }} \mathscr{F}_{a}^{(k)}$ and $\mathscr{F}_{h}^{(k)}$. We now have a sequence of extensions of $\mathscr{F}$

$$
\overline{\mathscr{F}}^{(k)} \stackrel{a_{k}}{\rightarrow} \mathscr{F}^{(k)} \hookrightarrow \mathscr{F}_{a}^{(k)} \hookrightarrow \mathscr{F}_{b}^{(k)},
$$

included in the following commutative diagram:

$$
\begin{aligned}
& \overline{\mathscr{F}}^{(k)} \stackrel{a_{k}}{\rightarrow} \mathscr{F}^{(k)} \hookrightarrow \mathscr{F}_{a}{ }^{(k)} \hookrightarrow \mathscr{F}_{b}^{(k)} \\
& \swarrow \quad \swarrow \quad \downarrow \downarrow \Pi \\
& \mathscr{X} \quad \mathscr{X}^{a} \quad \mathscr{X}^{b} \quad \mathscr{U}
\end{aligned}
$$

Given a locally free sheaf of $\mathcal{O}_{\mathscr{X}^{h}}$-modules $E_{\mathscr{X}^{h}}$, its restrictions $E_{\mathscr{X}}=\left.E_{\mathscr{X}^{b}}\right|_{\mathscr{X}}$ and $E_{\mathscr{X}^{a}}=\left.E_{X^{b}}\right|_{\mathscr{X}^{u}}$ arise. We shall consider the natural exact sequence of $\mathcal{O}_{\mathscr{X}^{b}}$-modules:

$$
0 \rightarrow \operatorname{ker} \mathscr{R} \rightarrow E_{\mathscr{X}^{h}} \stackrel{\mathscr{R}}{\rightarrow} E_{\mathscr{X}^{a}} \rightarrow 0,
$$

where $\mathscr{R}$ is the restriction map. Denote by $E_{a}^{(k)}$ the pull-back of $E_{\mathscr{X}^{u}}$ to $\mathscr{F}_{a}^{(k)}$, and by $E_{b}^{(k)}$ the pull-back of $E_{\mathscr{X}^{h}}$ to $\mathscr{F}_{h}^{(k)}$. On $\mathscr{F}_{b}^{(k)}$ we have a similar exact sequence

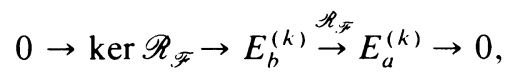

where $\mathscr{R}_{\mathscr{F}}$ is the restriction map corresponding to the embedding $\mathscr{F}_{a}{ }^{(k)} \hookrightarrow \mathscr{F}_{b}{ }^{(k)}$. 
On $\mathscr{U}$ we shall be interested in the direct image of sequence (8) under the morphism of analytic spaces $\Pi: \mathscr{F}_{b}{ }^{(k)} \rightarrow \mathscr{U}$. This is a long exact sequence of $\mathcal{O}_{\mathcal{U}}$-modules. If, for example, $\Pi$ is a proper morphism, this sequence will be a long exact sequence of coherent $\mathcal{O}_{\mathscr{U}}$-modules. Let us denote by $\Psi_{k}^{\prime}(a, b)$ the coboundary morphism $R^{\prime} \Pi_{*} E_{a}^{(k)} \rightarrow R^{\prime+1} \Pi_{*} \operatorname{ker} \mathscr{R}_{\mathscr{F}}$.

Looking for linear differential operators we are interested in the possible isomorphisms of terms of our long sequence of $\mathcal{O}_{\mathscr{U}}$-modules with some jet-bundles. We already know (see $\$ 2$ ) that the formal neighbourhoods $\check{\mathscr{F}}^{(k)}$ are responsible for the jet-bundles, and now we ask to what extent $\mathscr{F}_{a}{ }^{(k)}$ and $\mathscr{F}_{b}{ }^{(k)}$ have a similar property. But we have canonical morphisms $\overline{\mathscr{F}}^{(k)} \rightarrow \mathscr{F}_{a}{ }^{(k)}$ and $\overline{\mathscr{F}}^{(k)} \rightarrow \mathscr{F}_{b}{ }^{(k)}$ and this is the reason that under certain conditions one can obtain canonical isomorphisms from the type $R^{\prime} \Pi_{*} E_{a}^{(k)}=\mathscr{J}_{e} t^{m} E$ or $R^{l} \Pi_{*} E_{b}^{(k)}=\mathscr{J}_{e} t^{r} E$, where $E$ is some coherent sheaf on $\mathscr{U}$.

Let us now consider more explicity the special case when $\mathscr{X}^{a}=\mathscr{X}$, i.e. when only $\mathscr{X}^{\prime}$ is a nontrivial extension.

3.2. The case $\mathscr{X}^{a}=\mathscr{X}$. In this case the morphism $\overline{\mathscr{F}}^{(k)} \rightarrow \mathscr{F}_{a}{ }^{(k)}$ coincides with $a_{h}: \overline{\mathscr{F}}^{(k)} \rightarrow \mathscr{F}^{(k)}$ and we consider the exact sequence

$$
0 \rightarrow a_{k}^{-1}\left(p_{k}^{*} E_{\mathscr{X}}\right) \hookrightarrow P_{k}^{*} E_{\mathscr{X}} \rightarrow A_{k} \rightarrow 0,
$$

where $A_{k}$ is just the quotient of the first two sheaves. It is clear that $A_{k} \hookrightarrow$ $P_{h}^{*} E_{. \mathcal{Y}} \otimes r_{k-1}^{*}\left(\Omega_{\pi_{1}}^{1}\right)($ see 2.2$)$ and that the morphism $\Phi_{k-1}^{\prime}$ factors through $R^{\prime} q_{k *} A_{k}$ :

$$
\begin{gathered}
\Phi_{k-1}^{\prime}: R^{\prime} q_{k *} P_{k}^{*} E_{\mathscr{X}} \rightarrow R^{\prime} q_{k *}\left(P_{k}^{*} E_{\mathscr{X}} \otimes r_{k-1}^{*}\left(\Omega_{\pi_{1}}^{1}\right)\right) \\
\left(\begin{array}{cc}
\Phi_{k}^{\prime} & 1
\end{array}\right)^{\prime} \searrow \quad \nearrow\left(\Phi_{k-1}^{\prime}\right)^{\prime \prime} \\
R^{\prime} q_{k *} A_{k}
\end{gathered}
$$

Denote by $\delta_{k}^{l}$ the corresponding coboundary morphism

$$
\delta_{k}^{\prime}: R^{\prime} q_{k *} A_{k} \rightarrow R^{\prime+1} q_{k *} p_{k}^{*} E_{\mathscr{X}}
$$

If now $\left(\Phi_{k-1}^{\prime}\right)^{\prime}=0$ and $\delta_{k}^{\prime}=0$ (the vanishing of $R^{l-1} q_{k *} A_{k}$ and $R^{\prime} q_{k *} A_{k}$ is obviously sufficient), we obtain the desired isomorphism $R^{\prime} \Pi_{*} E_{a}^{(k)}=R^{\prime} q_{k} * p_{k}^{*} E_{\mathscr{X}}=$ $\mathscr{J}_{e} t^{k}\left(R^{\prime} \pi_{2 *} \pi_{1}^{*} E_{\mathscr{C}}\right)$. Thus the following theorem is true.

THEOREM. let $\mathscr{X}^{a}=\mathscr{X}$ and let $E_{\mathscr{X}^{b}}$ be a locally free sheaf on $\mathscr{X}^{b}$ such that $\left(\Phi_{k-1}^{\prime}\right)^{\prime}=0$ and $\delta_{k}^{\prime-1}=0$. Then $\Psi_{k}^{\prime}(a, b): R^{\prime} \Pi_{*} E_{a}^{(k)} \rightarrow R^{\prime+1} \Pi_{*} \operatorname{ker} R_{\mathscr{F}}$ determines a linear differential operator of order $k: \tilde{\Psi}_{k}^{l}(a, b): R^{\prime} \pi_{2 *} \pi_{1}^{*} E_{\mathscr{X}} \rightarrow R^{l+1} \Pi_{*} \operatorname{ker} \mathscr{R}_{\mathscr{F}}$.

REMARK. This Theorem shows that in contrast to $\Phi_{k-1}^{\prime}, \Psi_{k}^{\prime}(a, b)$ determine linear differential operators only when some cohomological conditions are satisfied. This is quite natural because even in the case when $\mathscr{X}^{a}=\mathscr{X}, \Psi_{k}^{\prime}(a, b)$ depends on the extension $\mathscr{X} \hookrightarrow \mathscr{X}^{b}$, which is a priori irrelevant to the geometry of $\mathscr{U}$.

It is remarkable that in this way we obtain a larger class of linear differential operators. 
Example. Let $\mathscr{U}=\operatorname{Gr}(2, T)$, where $T$ is a linear space and $\operatorname{dim} T=4$. Consider the diagram:

$$
\begin{gathered}
\mathscr{F}=\mathrm{Fl}(1,2,3, T) \\
\swarrow \quad \mathrm{P}(T) \times \mathbb{P}\left(T^{*}\right) \hookleftarrow \mathscr{X}=\mathrm{Fl}(1,3, T) \quad \operatorname{Gr}(2, T)=\mathscr{U} .
\end{gathered}
$$

Let $\mathscr{X}^{a}=\mathscr{X}$, and let $\mathscr{X}^{b}$ be the first formal neighbourhood of $\mathscr{X}$ in $\mathbb{P}(T) \times \mathbb{P}\left(T^{*}\right)$. Consider on $\mathbb{P}(T) \times \mathbb{P}\left(T^{*}\right)$ the bundle $\mathcal{O}(-2,0)$ (for $\mathcal{O}(0,-2)$ everything is absolutely analogous) and restrict it to $\mathscr{X}^{b}$. A cohomological computation shows that our construction leads to a surjective morphism of $\mathcal{O}_{\mathscr{U}}$-modules

$$
D_{+}: \mathscr{J}_{e} t^{2}\left(\Lambda^{2} S_{2}\right) \rightarrow\left(\Lambda^{2} S_{2}\right)^{\otimes 2} \otimes \Lambda^{2}\left(T / S_{2}\right)^{*} .
$$

By the fact that our diagram, together with the sheaf $\mathcal{O}(-2,0)$, is $\operatorname{GL}(T)$-invariant, $\tilde{D}_{+}$is also a $\operatorname{GL}(T)$-invariant differential operator and thus coincides with the conformally invariant Laplace operator on $\operatorname{Gr}(2, T)$ (see $[10,18])$ up to a constant factor. (Note that this operator could not be obtained as $\tilde{\Phi}_{k-1}^{l}$ for any bundle, because we consider an admissible $X$-structure and $\tilde{D}_{+}$is not a prolongation of a first order operator.)

3.3. The cases when both extensions $\mathscr{X}^{a}$ and $\mathscr{X}^{b}$ are nontrivial provide further interesting examples. Here we consider the simplest one-again the conformally invariant Dirac operator-and show the main steps of the computation.

Consider, as in the previous section, the diagram

$$
\begin{gathered}
\operatorname{Fl}(1,2,3, T) \\
\pi_{1} \swarrow \quad \downarrow \pi_{2} \\
\mathbb{P}(T) \times \mathbb{P}\left(T^{*}\right) \hookleftarrow \mathscr{X}^{b} \hookleftarrow \mathscr{X}^{a} \hookleftarrow \mathscr{X}=\operatorname{Fl}(1,3, T) \quad \operatorname{Gr}(2, T)
\end{gathered}
$$

but put $\mathscr{X}^{a}=\mathscr{X}^{(1)}$, and $\mathscr{X}^{b}=\mathscr{X}^{(2)}$ - the second neighbourhood of $\mathscr{X}$ in $\mathbb{P}(T) \times$ $\mathbb{P}\left(T^{*}\right)$. We begin with the sheaf $\mathcal{O}(-1,0)$ (or with $\mathcal{O}(0,-1)$ which evidently leads to a symmetric picture) on $\mathbb{P}(T) \times \mathbb{P}\left(T^{*}\right)$ and restrict it to $\mathscr{X}^{b}$. Consider now the sheaves $\mathcal{O}(-1,0)_{b}^{(2)}$ (on $\left.\mathscr{F}_{b}^{(2)}\right)$ and $\mathcal{O}(-1,0)_{a}^{(2)}$ (on $\mathscr{F}_{a}{ }^{(2)}$ ), which give rise to the following diagram on $\mathscr{F}_{b}^{(2)}$ :

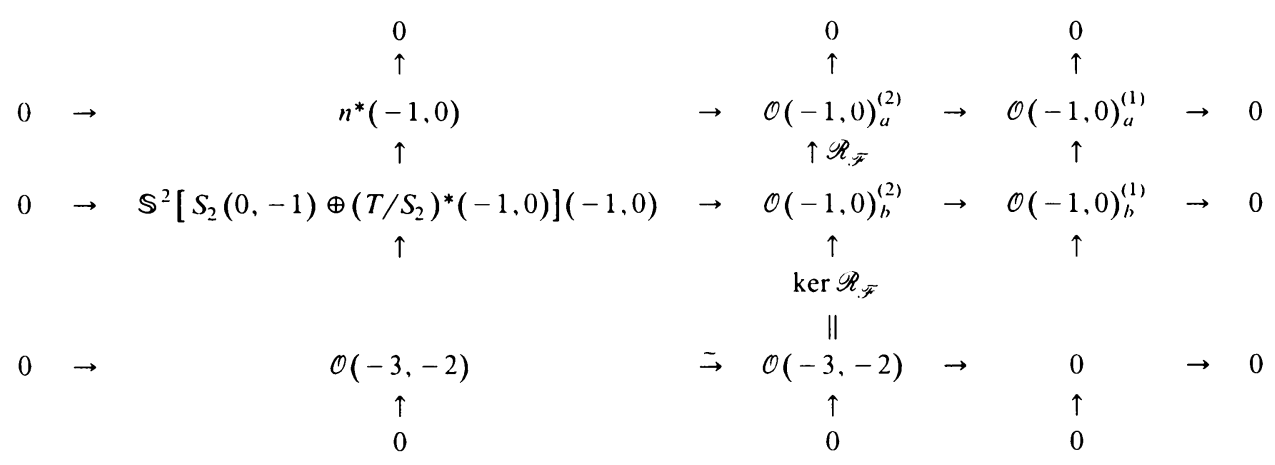

Here $S_{2}$ denotes the tautological bundle of rank 2 on $\mathscr{F}$ and $n^{*}(-1,0)$ is defined as the quotient of the two other sheaves of the left column. The terms of this diagram can be computed easily using the fact that the conormal bundle of $\mathscr{X}$ in $\mathbb{P}(T) \times \mathbb{P}\left(T^{*}\right)$ 
is $\mathcal{O}(-1,-1)$. The exact sequence (8) (for $k=2)$ is in our case the middle column. The right column coincides with sequence ( 8 ) for $k=1$, and we see that it reduces to an isomorphism. Apply the functor $R \Pi_{*}$ to this diagram. A direct cohomologicalcomputation shows that the direct image will have the form

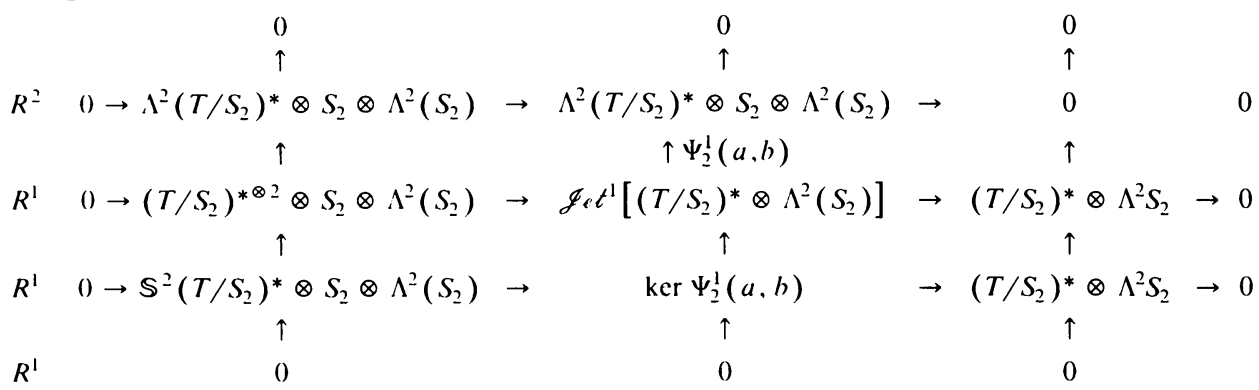

where on the left side we give the number of the corresponding term in the long exact cohomology sequence of the functor $R \Pi_{*}$. It is evident from the explicit calculation that $\Psi_{2}^{1}(a, b)$ is not zero because in the left column the corresponding morphism is not zero. (In the general case we also may look at the quotients in order to show that $\Psi_{k}^{\prime}(a, b)$ is not identically zero.) In our case $\tilde{\Psi}_{2}^{1}(a, b)$ is, of course, $\mathrm{GL}(T)$-invariant and therefore coincides with the Dirac operator (see [10]). Note that these operators slightly differ from the Dirac operators considered in 2.4.1. All four operators are natural prolongations of the two usual Dirac operators to the compact manifold $\operatorname{Gr}(2, T)$.

A similar construction may be carried out also for an arbitrary Grassmann $X$-structure.

REMARK. It seems quite interesting to study systematically the Penrose transform, i.e. the connection between $G$-invariant vector bundles and global $G$-invariant linear differential operators, in the special case when all spaces considered are flag spaces $G / P$ of a given reductive Lie group $G$.

3.4. How may we encode the operators $\Psi_{k}^{\prime}(a, b)$ directly in terms of the pair $\left(\mathscr{X}^{\prime}, E_{\mathscr{x}^{\prime}}\right)$ ? In [10 and 11] Henkin and Manin gave a description of the conformally flat Dirac and Laplace operators as coboundary operators in the cohomology of the sequence

$$
0 \rightarrow \operatorname{ker} \mathscr{R} \rightarrow E_{\mathscr{*}, h} \stackrel{\mathscr{R}}{\rightarrow} E_{\mathscr{Y}^{\prime \prime}} \rightarrow 0
$$

(this can be considered as the inverse Penrose transform applied to these operators). A curved version of these results for the Laplace operator is contained in [17].

Our construction enables us to understand under what conditions such a description is possible in the general case. Indeed, $\Psi_{k}^{l}(a, b)$ is the coboundary morphism

$$
R^{\prime} \Pi_{*} E_{a}^{(k)} \rightarrow R^{\prime+1} \Pi_{*} \operatorname{ker} \mathscr{R}_{\bar{\pi}},
$$

and if the Leray spectral sequence of $E_{a}^{(k)}$ under the morphism $\Pi$ degenerates $\Psi_{k}^{\prime}(a, b)$ can be encoded in the cohomology of sequence (8) on $\mathscr{F}_{b}{ }^{(k)}$ :

$$
\Psi_{k}^{\prime}(a, b): H^{\prime}\left(\mathscr{F}_{a}{ }^{(k)}, E_{a}^{(k)}\right) \rightarrow H^{\prime+1}\left(\mathscr{F}_{b}^{(k)}, \operatorname{ker} \mathscr{R}_{\mathscr{F}}\right) .
$$


Further, we suppose that this is satisfied. We have also the projection $\mathscr{F}_{b}^{(k)} \rightarrow \mathscr{X}^{b}$ and therefore it is natural to ask whether we can construct the following commutative diagram:

$$
\begin{aligned}
& H^{0}\left(\mathscr{U}, \mathscr{J}_{e} t^{m} E\right)=H^{\prime}\left(\mathscr{F}_{a}{ }^{(k)}, E_{a}^{(k)}\right) \rightarrow H^{l+1}\left(\mathscr{F}_{b}{ }^{(k)}, \operatorname{ker} \mathscr{R}_{\mathscr{F}}\right) \\
& \uparrow j_{m} \quad \| \\
& H^{0}(\mathscr{U}, E)=H^{\prime}\left(\mathscr{X}^{a}, E_{\mathscr{y}^{a}}\right) \stackrel{\tilde{\Psi}_{h}^{\prime}(a, h)}{\rightarrow} H^{l+1}\left(\mathscr{X}^{h}, \operatorname{ker} \mathscr{R}\right)
\end{aligned}
$$

i.e. can we encode $\tilde{\Psi}_{k}^{\prime}(a, b)$ as the lower row of this diagram?

The equality in the right column of the diagram is a necessary cohomological condition, which we have to assume being satisfied, so let us turn to the other part of the diagram.

Note that we always have a map $H^{\prime}\left(\mathscr{X}^{a}, E_{\mathscr{X}^{a}}\right) \rightarrow H^{\prime}\left(\mathscr{F}_{a}{ }^{(k)}, E_{a}^{(k)}\right)$ (the left column of the diagram) and the point is that it can be identified with $j_{m}$ when some further cohomological restrictions are satisfied. Let us, for example, write down these conditions for the special case when $\mathscr{X}^{a}=\mathscr{X}$. We suppose first, as in the Theorem of 3.2, that $\left(\Phi_{k-1}^{\prime}\right)^{\prime}=0$ and $\delta_{k}^{l-1}=0$ (so that $\Psi_{k}^{\prime}(a, b)$ determines a differential operator). Then $E=R^{\prime} \pi_{2 *} \pi_{1}^{*} E_{\mathscr{X}}$ and $R^{\prime} \Pi_{*} E_{a}^{(k)}=\mathscr{J}_{e} t^{k} E=R^{\prime} Q_{k *} P_{k}^{*} E_{\mathscr{X}}$.

If now the Leray spectral sequence of $P_{k}^{*} E_{\mathscr{X}}$ under $Q_{k} *$ degenerates, we obtain that $H^{0}\left(\mathscr{U}, \mathscr{J}_{e} t^{k} E\right)=H^{\prime}\left(\overline{\mathscr{F}}^{(k)}, P_{k}^{*} E_{\mathscr{X}}\right)$. Postulating similarly, $H^{0}(\mathscr{U}, E)=$ $H^{\prime}\left(\mathscr{X}, E_{\mathscr{X}}\right)=H^{\prime}\left(\mathscr{F}, \pi_{1}^{*} E_{\mathscr{X}}\right)$ (this will be satisfied if $\pi_{1}^{-1}$ induces an isomorphism between the cohomology of $E_{\mathscr{X}}$ and $\pi_{1}^{-1}\left(E_{\mathscr{X}}\right)$ and if $H^{l-1}\left(E_{\mathscr{F}} / \pi_{1}^{-1}\left(E_{\mathscr{X}}\right)\right)=$ $H^{\prime}\left(E_{\bar{T}} / \pi_{1}^{-1}\left(E_{\mathscr{X}}\right)\right)=0$ ) we obtain our desired diagram because now the map

$$
H^{0}(\mathscr{U}, E) \rightarrow H^{0}\left(\mathscr{U}, \mathscr{J}_{e} t^{k} E\right)
$$

coincides with the natural map

$$
\Xi: R^{\prime} \pi_{2 *} \pi_{1}^{*} E_{\mathscr{U}} \rightarrow R^{\prime} Q_{k} * r_{k}^{*} \pi_{1}^{*} E_{\mathscr{U}}=\mathscr{J}_{e} t^{k} E
$$

induced by the diagram:

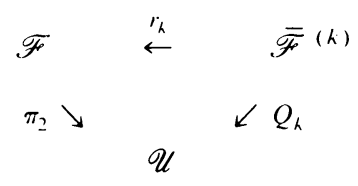

But as we know from the proof of the Theorem in 2.3 (Lemma 2) $\Xi$ is canonically identified with $j_{k}$, and thus $\tilde{\Psi}_{k}^{\prime}(a, b)$ coincides with the coboundary morphism $\delta^{\prime}$ in the long exact sequence associated to sequence (7) on $\mathscr{X}^{h}$.

These considerations are only an illustration of the fact that the vanishing of sufficiently many cohomology groups (all our conditions can be reformulated this way) automatically implies that $\tilde{\Psi}_{k}^{\prime}(a, b)$ is a coboundary operator in the cohomology of $t^{b}$.

The corresponding computations, which prove the results of Henkin and Manin are quite straightforward and thus we omit them. 


\section{REFERENCES}

1. M. F. Atiyah, Geometry of Yang-Mills fields, Lezioni Fermiane, Pisa, 1979.

2. M. F. Atiyah, N. J. Hitchin, V. G. Drinfeld and Yu. I. Manin, Construction of instantons, Phys. Lett. A 65 (1978), 185-187.

3. N. P. Buchdahl, Analysis on analytic spaces and non-self-dual Yang-Mills fields, preprint, Oxford Univ. Press, New York and London, 1982.

4. On the relative de Rham sequence, preprint, Oxford Univ. Press, New York and London, 1982.

5. P. Deligne, Equationes differentielles à points singuliers réguliers, Lecture Notes in Math., vol. 163, Springer-Verlag, Berlin and New York, 1970.

6. M. Eastwood, R. Penrose and R. O. Wells, Jr., Cohomology and massless fields, Comm. Math. Phys. 78 (1981), 305-351.

7. H. Grauert, Ein Theorem der analytischen Garhentheorie und die Modulräume komplexer Strukturen, Inst. Hautes Études Sci. Publ. Math. 5 (1960), 233-292.

8. Ph. A. Griffiths, The extension problem in complex analysis. II, Amer. J. Math. 88 (1966), 366-446.

9. R. Hartshorne, Algebraic geometry, Graduate Texts in Math., no. 52, Springer-Verlag, Berlin and New York, 1977.

10. (i. M. Henkin and Yu. I. Manin, Twistor description of classical Yang-Mills-Dirac fields, Phys. Lett. B 95 (1980), 405-408.

11. On the cohomology of twistor flag spaces, Compositio Math. 44 (1981), 103-111.

12. N. J. Hitchin, Monopoles and geodesics, Comm. Math. Phys. 83 (1982), 579-602.

13. On the construction of monopoles, Comm. Math. Phys. 89 (1983), 145-190.

14. J. Isenberg and Ph. Yasskin, Twistor description of non-self-dual Yang-Mills fields, preprint, Univ. of Marvland, 1978 .

15. J. Isenberg, Ph. Yasskin and P. Green, Non-self-dual gauge fields, Phys. Lett. B 78 (1978), 462-464.

16. C. R. Le Brun, Spaces of complex geodesics and related structures, Ph.D. Thesis, Oxford, 1980.

17. The first formal neighbourhood of ambitwistor space for curved space-time, preprint, Inst. Hautes Études Sci., 1981.

18. A. Douady and T. L. Verdier, Les equations de Yang-Mills, Asterisque 71-72 (1980).

19. Yu. I. Manin, Gauge fields and complex geometri, Plenum Press, New York (to appear).

20. I. B. Penkov, The Penrose transform on general Grassmannians, C. R. Acad. Bulgare Sci. 33 (1980), 14.39-1442.

21. R. Penrose, Nonlinear gravitons and curted twistor theory, Gen. Relativity Gravitation 7 (1976), $31-52$.

22. __ The twistor program, Rep. Math. Phys. 12 (1977), 65-76.

23. D. C. Spencer, Overdetermined s!stems of linear partial differential equations, Bull. Amer. Math. Soc. 75 (1969), 179-239.

24. R. Ward, On self-dual gauge fields, Phys. Lett. A 61 (1977), 81-82.

25. R. O. Wells, Jr., Complex manifolds and mathematical phisics, Bull. Amer. Math. Soc. (N. S.) 1 (1979), 296-336.

26. $567-600$.

, Hyperfunction solutions of the zero-rest-mass field equation, Comm. Math. Phys. 78 (1981),

27. E. Witten, An interpretation of classical Yang-Mills theory, Phys. Létt. 77 B (1878), 394-402.

28. С. Г. Гиндикин и Г. М. Хенкин, Преобразование Пенроуза и комплексная интегральная геометрия, ВИНИТИ, Совр. пробл. мат. 17 (1981), 57-111.

29. В. Г. Дринфельд и Ю. И. Манин, Инстантоны и пучки на $\mathrm{CP}^{3}$, Функц. анализ и его прил., 13 вып. 2 (1979), 59-74.

30. Ю. И. Манин, Калибровочные поля и голоморфная геометрия, ВИНИТИ, Совр. пробл. мат. 17 (1981), 3-56.

31. __ Голоморфная геометрия и суперполя Янга-Миллса, ВИНИТИ, Совр. пробл. мат. 24 (1984), 3-80.

32. И. Б. Пенков, Линейные дифференциальные операторы и когомологии аналитических пространств, УМН, том 37, вып. 4(226), (1982), 171-172.

Institute of Mathematics, P.O. Box 373, 1090 Sofia, Bulgaria

Current address: V. A. Steklov Institute, ul. Vavilova 42, 11733 Moscow, USSR 